Cite this: Phys. Chem. Chem. Phys., 2012, 14, 13933-13948

\title{
Kinetics and mechanism of the reaction of $\mathrm{OH}$ with the trimethylbenzenes - experimental evidence for the formation of adduct isomers $\dagger$
}

\author{
Birger Bohn ${ }^{a}$ and Cornelius Zetzsch ${ }^{b c}$ \\ Received 17th July 2012, Accepted 15th August 2012 \\ DOI: $10.1039 / \mathrm{c} 2 \mathrm{cp} 42434 \mathrm{~g}$
}

The reversible gas-phase addition of $\mathrm{OH}$ radicals to the trimethylbenzenes was investigated in pulsed experiments utilizing VUV flash-photolysis resonance-fluorescence of $\mathrm{H}_{2} \mathrm{O}$ in the temperature range of $275-340 \mathrm{~K}$. Triexponential $\mathrm{OH}$ decays were observed in the presence of the trimethylbenzenes, indicating the participation of more than one adduct species. Analytical solutions for the system of differential equations with two adduct isomers were derived, and the $\mathrm{OH}$ decay curves were evaluated based on this reaction model. This led to significant improvements of fit qualities and notable changes in $\mathrm{OH}$ rate constants compared to a previous model with a single adduct species. The detailed analysis was confined to 1,3,5-trimethylbenzene where reversible formation of two $\mathrm{OH}$-aromatic ortho- and ipso-adduct isomers is feasible in accordance with the extended reaction model. Only after inclusion of additional isomerization reactions, consistent thermochemical data were obtained from the fitted rate constants.

Reaction enthalpies of $-83 \pm 7 \mathrm{~kJ} \mathrm{~mol}^{-1}$ and $-35 \pm 22 \mathrm{~kJ} \mathrm{~mol}^{-1}$ were derived for the formation of one adduct isomer and the isomerization into the other, respectively. Based on literature data, the more and less stable adducts were assigned to ipso- and ortho-adduct isomers, respectively. The potential isomerization precluded the determination of primary yields of adduct isomers but formation of the ipso-adduct in any case is a minor process. For the rate constants of the $\mathrm{OH}+1,3,5$-trimethylbenzene reaction an Arrhenius expression $k_{\mathrm{OH}}=1.32 \times 10^{-11} \mathrm{~cm}^{3} \mathrm{~s}^{-1}$ $\exp (450 \pm 50 \mathrm{~K} / T)$ was obtained. Based on the same approach, the rate constants of the $\mathrm{OH}$ reactions with 1,2,3-trimethylbenzene and 1,2,4-trimethylbenzene were derived as $k_{\mathrm{OH}}=3.61 \times 10^{-12} \mathrm{~cm}^{3} \mathrm{~s}^{-1} \exp (620 \pm 80 \mathrm{~K} / T)$ and $k_{\mathrm{OH}}=2.73 \times 10^{-12} \mathrm{~cm}^{3} \mathrm{~s}^{-1}$ $\exp (730 \pm 70 \mathrm{~K} / T)$, respectively.

\section{Introduction}

Aromatic compounds are important anthropogenic polluters of the urban atmosphere affecting air quality by secondary formation of ozone and particulate matter. The gas-phase degradation of aromatics under atmospheric conditions is mainly initiated by reactions with $\mathrm{OH}$ radicals followed by secondary reactions with $\mathrm{O}_{2}$ leading to ring-cleavage products (unsaturated carbonyl compounds, glyoxals and epoxides) or oxidized ring-retaining products (phenols, benzaldehydes). ${ }^{1-3}$ The initial steps of the oxidation processes have been studied

${ }^{a}$ Institut für Energie- und Klimaforschung IEK-8: Troposphäre, Forschungszentrum Jülich GmbH, 52425 Jülich, Germany.

E-mail: b.bohn@fz-juelich.de; Fax: +492461615346

${ }^{b}$ Atmospheric Chemistry Research Laboratory, University of Bayreuth, 95448 Bayreuth, Germany

${ }^{c}$ Fraunhofer Institute for Toxicology and Experimental Medicine, 30625 Hannover, Germany

$\dagger$ Electronic supplementary information (ESI) available. See DOI: $10.1039 / \mathrm{c} 2 \mathrm{cp} 42434 \mathrm{~g}$ for a number of aromatic compounds under laboratory and simulation chamber conditions. However, many details regarding the product yields of the different reaction pathways are still uncertain.

The initial $\mathrm{OH}$ reaction mainly proceeds by addition, forming an OH-aromatic adduct. Owing to the stability of the aromatic ring this adduct is unstable and the addition is markedly reversible, at least above room temperature. Although the reversibility is unimportant in the atmosphere because of fast competing reactions, by observing $\mathrm{OH}$ in equilibrium with the adduct kinetic information on the formation and fate of the adduct can be derived which is of fundamental interest in understanding the oxidation mechanisms of aromatic compounds. For example, in experiments with pulsed $\mathrm{OH}$ formation in the presence of aromatics, the reversible addition leads to complicated $\mathrm{OH}$ decay curves. ${ }^{4-7}$ In previous studies these $\mathrm{OH}$ decay curves were analysed in terms of a kinetic model assuming that a single adduct species is formed as in the case of benzene. The corresponding analytical solution then 
predicts biexponential $\mathrm{OH}$ decay curves (sum of two exponential decays) that were fitted to the experimental data to extract rate constants for reactions of both $\mathrm{OH}$ and the adduct. ${ }^{4,5}$ Moreover, because data analysis of biexponential decay curves is not as straightforward as for monoexponential decays, advanced tools were developed that allowed us to fit whole sets of curves obtained at different reactant concentrations simultaneously. This method was applied successfully for a number of aromatic compounds. ${ }^{6,7} \mathrm{An}$ important finding was that the adduct $+\mathrm{O}_{2}$ reactions are the dominant secondary reactions under typical atmospheric conditions. ${ }^{6,7}$

However, except for a few selected compounds, the assumption of a single adduct species is a simplification. Typically several adduct isomers can be formed. For example, for the series of methyl-substituted monocyclic aromatic compounds carrying one to six $\mathrm{CH}_{3}$-groups, only hexamethylbenzene is expected to form strictly one adduct isomer, an ipso-adduct in this case. ${ }^{7}$ All other compounds can form two or more structural isomers with a maximum of six in the case of 1,2,4-trimethylbenzene, not considering stereoisomers with identical thermochemical properties. In principle this is expected to lead to highly complicated, multiexponential $\mathrm{OH}$ decay curves. Nevertheless, $\mathrm{OH}$ decay curves mostly turned out to be effectively biexponential within experimental error. This can be explained by similar properties of different isomers, a dominant formation of one isomer, or fast isomerizations. An example is toluene where no deviation from biexponential behaviour was observed ${ }^{6}$ although four possible isomers can be formed by $\mathrm{OH}$ addition at ortho-, meta-, para-, and ipsopositions with respect to the $\mathrm{CH}_{3}$-group. More recently we noticed deviations from biexponential behaviour in the case of 1,3,5-trimethylbenzene in the data set examined in this work, but no quantitative evaluation was attempted. ${ }^{7}$

Before about ten years ago, ipso-type adducts, where the $\mathrm{OH}$ adds at an already substituted position at the aromatic ring, were hardly considered in the gas-phase reaction mechanism. At that time theoretical work predicted significant yields for the ipso-adduct of toluene. ${ }^{8}$ Moreover, the rate constant of the $\mathrm{OH}+$ hexamethylbenzene reaction was shown to be extremely fast and inconsistent with the expected, slow rate constant for the H-atom abstraction reaction from the $\mathrm{CH}_{3}$-substituent groups. ${ }^{9}$ Reversible formation of an adduct in the $\mathrm{OH}+$ hexamethylbenzene reaction was confirmed in the meantime and the adduct $+\mathrm{O}_{2}$ reaction was studied by the method outlined above. ${ }^{7}$ The latter two studies established the existence of ipsotype adducts experimentally, at least for the fully substituted hexamethylbenzene. As a consequence, the number of potentially significant isomers increased for substituted aromatic compounds but the actual yields of different isomers remain widely unknown.

The measurements with trimethylbenzenes (TMB) analysed in this work were already made several years ago. A former analysis based on the usual assumption of one adduct species was not satisfactory and for that reason the results were not published previously with the exception of the rate constants of the $\mathrm{OH}+\mathrm{TMB}$ reactions for the three isomers 1,3,5-, 1,2,3and 1,2,4-TMB that were released in the form of temperature dependent parameterizations. ${ }^{10}$ These results are revised in this work. The possibility that adduct isomers were responsible for the problems with the data analysis was not considered in the former evaluation because at least for the symmetric 1,3,5TMB no adduct isomers were expected neglecting formation of ipso-adducts. Moreover, the concept of considering more than one adduct isomer in the analysis had not yet been developed.

In this work an extended kinetic model considering two adduct isomers will be introduced and applied in a re-analysis of the previously obtained experimental data with $1,3,5-\mathrm{TMB}^{10}$ based on analytical solutions. We will show that this leads to an improved description of the data consistent with formation of two adduct isomers. The same procedure was also applied to the previously obtained data with 1,2,3-TMB and 1,2,4-TMB to correct the $\mathrm{OH}$ rate constants. ${ }^{10}$ Although the number of possible adduct isomers is greater than two for these compounds, the improvement of data description by the extended model was comparable, justifying this approach.

\section{Experimental}

The flash-photolysis/resonance fluorescence (FP/RF) apparatus used in this work is based on work by Stuhl and Niki ${ }^{11,12}$ and subsequent developments. ${ }^{4,5} \mathrm{~A} \mathrm{FP} / \mathrm{RF}$ cell with efficient, antireflection coated quartz optics, partly automated pulsed photolysis, as well as digital data acquisition and evaluation by a programmable microprocessor (FLEXTRAN, Tracor, and LSI 11/2, Digital Equipment Corporation) was developed by Wahner and Zetzsch. ${ }^{4}$ The FP/RF cell with similar optics and dimensions but variable temperature, used in the present study, has been constructed and employed by Witte $e t a l .{ }^{5}$ The RF sensitivity was further improved by placing the microwave discharge into the focus of the optics, and convenient software was developed for automated controlling and monitoring of the whole experiment (flash lamp, electronic mass flow controllers, magnetic valves, temperature, total pressure, partial pressures of water as a precursor of $\mathrm{OH}$ and reactant). A detailed description of the setup was given by Koch et al. ${ }^{7}$

$\mathrm{OH}$ radicals were produced in helium buffer gas by pulsed VUV flash-photolysis of water vapour in a thermostated reaction volume. $\mathrm{OH}$ was continuously excited electronically by $\mathrm{OH}$ fluorescence around $308 \mathrm{~nm}$ from an attached microwave discharge lamp running with argon and added water vapour. After passing an interference filter, fluorescence from the reaction volume was detected by a photomultiplier mounted at right angles to the flash lamp and the resonance lamp. Photomultiplier signals were recorded using the photon counting technique with a multichannel scaler board. Typically 150-300 single experiments were accumulated to obtain sufficient intensity for a proper evaluation of the decay curves. Background was recorded for up to $5 \mathrm{~s}$ and pulse repetition rates were $0.2 \mathrm{~Hz}$. Background count rates ranged around $40 \mathrm{kHz}$ while typical $\mathrm{OH}$ starting count rates were $50 \mathrm{kHz}$. With 150-300 single measurements this resulted in $S / N$-ratios of $70-100$ at starting interval widths of $1.2 \mathrm{~ms}$.

In order to remove high-frequency noise and avoid unnecessary large data files, the data originally recorded by the multichannel scaler were re-binned using interval widths that increased with reaction time following a verified algorithm. ${ }^{7,13}$ By this data compression the original 4096 data points were condensed to 61 values for each decay curve that were roughly equidistant 
Table 1 Summary of experimental conditions and biexponential model-1 fit results for experiments with 1,3,5-TMB. Left: Temperatures $T$, total pressures $p$ of $\mathrm{He}$, range of reactant concentrations, and the number $m$ of $\mathrm{OH}$ decay curves recorded. Right: Model-1 fit results and estimated error limits from simultaneous fits to the $m$ decay curves at different aromatics concentrations

\begin{tabular}{|c|c|c|c|c|c|c|c|c|c|c|}
\hline \# & $T / \mathrm{K}$ & $p / \mathrm{hPa}$ & [Aromatic] $/ 10^{12} \mathrm{~cm}^{-3}$ & $m$ & $k_{2} / \mathrm{s}^{-1}$ & $k_{1 \mathrm{a}}+k_{1 \mathrm{~b}} / 10^{-11} \mathrm{~cm}^{3} \mathrm{~s}^{-1}$ & $k_{1 \mathrm{a}} k_{-1 \mathrm{a}} / 10^{-10} \mathrm{~cm}^{3} \mathrm{~s}^{-2}$ & $k_{-1 \mathrm{a}}+k_{3} / \mathrm{s}^{-1}$ & DOF & $\chi^{2} / \mathrm{DOF}$ \\
\hline 1 & 276.3 & 380 & $1.0-5.7$ & 6 & $19.6 \pm 6.1$ & $6.82 \pm_{0.40}^{0.42}$ & $0.43 \pm \begin{array}{l}0.47 \\
0.25\end{array}$ & $4.2 \pm{ }_{3.3}^{11}$ & 350 & 1.17 \\
\hline 2 & 282.8 & 380 & $1.0-5.7$ & 10 & $16.2 \pm 4.8$ & $6.54 \pm 0.37$ & $0.81 \pm 0.27$ & $4.5 \pm{ }_{1.5}^{2.1}$ & 586 & 1.11 \\
\hline 3 & 288.1 & 750 & $1.0-6.7$ & 7 & $10.4 \pm 6.0$ & $6.40 \pm 0.43$ & $1.20 \pm 0.46$ & $5.1 \pm{ }_{1.8}^{2.9}$ & 409 & 1.16 \\
\hline 4 & 293.4 & 750 & $1.0-5.7$ & 6 & $9.1 \pm 4.1$ & $6.11 \pm 0.31$ & $1.75 \pm 0.30$ & $6.3 \pm 1.2$ & 350 & 1.06 \\
\hline 5 & 298.9 & 380 & $0.9-5.8$ & 7 & $14.5 \pm 2.8$ & $5.77 \pm 0.24$ & $2.48 \pm 0.22$ & $6.4 \pm 0.5$ & 409 & 1.60 \\
\hline 6 & 299.1 & 750 & $0.5-5.3$ & 7 & $9.8 \pm 1.1$ & $5.76 \pm 0.20$ & $2.57 \pm 0.21$ & $7.1 \pm 0.5$ & 409 & 1.57 \\
\hline 7 & 304.4 & 380 & $0.9-5.7$ & 7 & $10.2 \pm 1.8$ & $5.62 \pm{ }_{0.22}^{0.23}$ & $4.16 \pm{ }_{0.28}^{0.30}$ & $10.0 \pm{ }_{0.5}^{0.6}$ & 409 & 1.79 \\
\hline 8 & 313.9 & 380 & $0.9-5.5$ & 7 & $9.2 \pm 1.0$ & $5.03 \pm 0.26$ & $8.44 \pm \begin{array}{l}0.70 \\
0.65\end{array}$ & $19.9 \pm 0.9$ & 409 & 2.98 \\
\hline 9 & 318.4 & 750 & $0.5-5.3$ & 7 & $8.0 \pm 0.5$ & $5.18 \pm 0.37$ & $13.6 \pm 1.6$ & $30.6 \pm 1.7$ & 409 & 2.97 \\
\hline 10 & 323.5 & 380 & $0.5-5.2$ & 7 & $5.5 \pm 0.3$ & $4.62 \pm 0.51$ & $17.8 \pm 2.7$ & $43.6 \pm 3.0$ & 409 & 2.62 \\
\hline 11 & 333.1 & 380 & $0.5-5.2$ & 7 & $5.0 \pm 0.3$ & $3.65 \pm \begin{array}{l}0.76 \\
0.65\end{array}$ & $28.2 \pm 9.7$ & $86.8 \pm 9.1$ & 409 & 2.60 \\
\hline 12 & 333.1 & 750 & $0.5-5.2$ & 7 & $4.9 \pm 0.2$ & $3.94 \pm 0.75$ & $32.9 \pm 7.9$ & $93.0 \pm 9.8$ & 409 & 1.81 \\
\hline 13 & 338.4 & 750 & $0.5-5.3$ & 7 & $5.1 \pm 0.2$ & $3.69 \pm \begin{array}{l}0.82 \\
0.69\end{array}$ & $46.4 \pm 17$ & $140 \pm \begin{array}{l}15 \\
15\end{array}$ & 409 & 2.95 \\
\hline 14 & 340.0 & 380 & $0.5-5.1$ & 7 & $5.3 \pm 0.2$ & $2.88 \pm \begin{array}{l}1.04 \\
0.80\end{array}$ & $33.4 \pm 21$ & $132 \pm 25$ & 409 & 4.20 \\
\hline
\end{tabular}

on a logarithmic time scale. It was tested that this procedure led to no systematic deviations in the subsequently applied curve fitting procedures, ${ }^{7}$ but the variable interval width had to be considered explicitly in the data analysis.

Experimental conditions for 1,3,5-trimethylbenzene are summarized in Table 1. Typically seven $\mathrm{OH}$ decay curves at different reactant concentrations were recorded for each temperature. Temperatures ranged between $275 \mathrm{~K}$ and $340 \mathrm{~K}$. Using calibrated mass flow controllers, the gas-mixture was slowly flowing through the reaction volume to avoid build-up of reaction and photolysis products. Helium was used as a buffer gas to minimize quenching of excited $\mathrm{OH}$. Total pressures of $380 \mathrm{hPa}$ and $750 \mathrm{hPa}$ were employed at total volume flow rates of 1000 and $2000 \mathrm{sccm}$, respectively. The gas-mixture was entering the reaction cell through a thermostated Woods horn (acting as a radiation trap opposite to the resonance lamp). This resulted in a reduction of flow velocities and effective heat exchange with the thermostated walls of the reaction cell before the gas mixture reached the detection volume. Flow velocities below $1 \mathrm{~cm} \mathrm{~s}^{-1}$ were evaluated for the detection volume.

$\mathrm{OH}$ starting concentrations were estimated based on previous work ${ }^{7,14}$ and were around $1 \times 10^{10} \mathrm{~cm}^{-3}$ at $\mathrm{H}_{2} \mathrm{O}$ concentrations of typically $4 \times 10^{15} \mathrm{~cm}^{-3}$. Because the $\mathrm{OH}$ precursor $\mathrm{H}_{2} \mathrm{O}$ is unreactive towards $\mathrm{OH}$, experiments were feasible at relatively long time-scales. OH was detectable for up to $1 \mathrm{~s}$, dependent on experimental conditions. Consequently, also low concentrations of added reactants were sufficient $\left(<6 \times 10^{12} \mathrm{~cm}^{-3}\right)$ but pseudo-first order conditions always applied for $\mathrm{OH}$ in the presence of the aromatic reactants. Aromatics were introduced by a gas saturation technique where a known flow of buffer gas was fed through a liquid sample of the reactant taking up the vapour pressure at an accurately known temperature of typically $260 \mathrm{~K}$. Vapour pressures of the TMB reactants were calculated using parameterizations from the literature ${ }^{15}$ with a stated $4.8 \%$ accuracy. For 1,3,5-trimethylbenzene the vapour pressures are confirmed within $2 \%$ around $260 \mathrm{~K}$ in a more recent compilation. ${ }^{16}$ We further estimate a $\pm 0.3 \mathrm{~K}$ accuracy of our temperature measurements that translate to about $5 \%$ additional uncertainties of the resulting aromatics concentrations.
Decay curves in the absence of aromatics were not regularly recorded during the measurements because turning on and off of the gas saturation system induced too long waiting times. Test measurements with no added aromatics showed that the pressure and temperature dependence of the background decay rate constants was consistent with those that were extrapolated from the measurements with added aromatics (Section 3.3). However, a minor complication was that $\mathrm{OH}$ decays with no added aromatics to some extent remained biexponential most likely because part of the background reactivity was attributable to aromatic compounds. A quantitative explanation of this behaviour is difficult. On the other hand, this unaccounted, slow recycling of $\mathrm{OH}$ is negligible upon addition of aromatics when the total $\mathrm{OH}$ reactivity was dominated by the added reactants.

Because 1,3,5-TMB is strongly absorbing in a wavelength range below $230 \mathrm{~nm}$, the fraction that can be photolyzed was estimated from a measured spectrum of the flash-lamp. ${ }^{17}$ The spectral range considered was limited to $>150 \mathrm{~nm}$ because the cut-off of the quartz lens used was around $160 \mathrm{~nm}$ and to $<290 \mathrm{~nm}$ because neither $\mathrm{H}_{2} \mathrm{O}$ nor 1,3,5-TMB dissociate or absorb in that region. Based on absorption cross sections of $\mathrm{H}_{2} \mathrm{O}$ from the literature, ${ }^{18}$ the spectrum was scaled to match a $\mathrm{OH}$ starting concentration of $1 \times 10^{10} \mathrm{~cm}^{-3}$. A photolyzed fraction of $1,3,5-\mathrm{TMB}<1 \times 10^{-4}$ was then obtained using absorption cross sections of 1,3,5-TMB from the literature ${ }^{19,20}$ and a maximum quantum yield of unity. Thus, even if the potential photo-fragments, e.g. dimethylbenzyl radicals, are highly reactive towards $\mathrm{OH}$ they can hardly compete with the large excess of 1,3,5-TMB, a quite reactive compound by itself.

Nevertheless, radical-radical reactions following the initial formation of $\mathrm{OH}$ radicals and $\mathrm{H}$-atoms in the $\mathrm{H}_{2} \mathrm{O}$ photolysis may contribute to the final loss of $\mathrm{OH}$ and $\mathrm{OH}$-aromatic adducts from the detection volume. Because radical concentrations were low, these processes were not considered explicitly and were assumed to be incorporated in first-order background loss rate constants of all radical species. The same applies for diffusion processes. Based on numerical simulations we will show that this approach is justified (Section 3.3).

The purity of the helium was $99.996 \%$ (Messer). Traces of oxygen were removed with Oxisorb cartridges (Messer). Double distilled water was introduced by a second gas 
saturation system. The purity of the aromatic reactants was checked by gas-chromatographic analysis (50 m capillary column and flame ionization detector). 1,3,5-TMB (Fluka, $99+$ ) had a purity of $99.4 \%$ and contained $0.6 \%$ of $1,2,4-$ TMB. 1,2,4-TMB (Fluka, 99+) was found to be $98.9 \%$ pure and contained $0.6 \%$ of 2-ethyltoluene, $0.3 \%$ of $1,2,3-$ TMB and $0.2 \%$ of 1,3,5-TMB. 1,2,3-TMB was available only with technical grade specification (Aldrich, $>90 \%$ ) but was found to have a purity of $94.2 \%$ and to contain $2.3 \%$ of $1,2,4-\mathrm{TMB}, 0.2 \%$ of $1,3,5-\mathrm{TMB}$ and $3.3 \%$ of ethyldimethylbenzenes.

\section{Results and discussion}

\subsection{Reaction models and analytical solutions}

3.1.1 Formation of one adduct species - model-1. The basic kinetic model of adduct formation and the corresponding analytical solutions will be summarized here briefly for direct comparison with the extended approach. Moreover, we derived more general expressions than previously that may also be applied at different boundary conditions and for other chemical systems (Section S1, ESI $\dagger$ ).

$\mathrm{OH}$ radicals are assumed to react with aromatic compounds under reversible formation of a single adduct (add):

$$
\mathrm{OH}+\operatorname{aromatic} \rightleftharpoons \operatorname{add}\left(k_{1 \mathrm{a}}, k_{-1 \mathrm{a}}\right) \quad(\mathrm{R} 1 \mathrm{a} /-1 \mathrm{a})
$$

The corresponding first- and second-order rate constants are defined in brackets. Other, irreversible reactions are also possible, in particular abstraction reactions for substituted aromatics at higher temperatures:

$$
\mathrm{OH}+\text { aromatic } \rightarrow \text { products }\left(k_{1 \mathrm{~b}}\right)
$$

$\mathrm{OH}$ and the adduct may also react with impurities, by wall loss, or vanish from the observation zone by diffusional spread. These processes are usually minor, independent of the aromatics concentration and accounted for by two further hypothetical reactions:

$$
\begin{aligned}
& \mathrm{OH} \rightarrow \text { products }\left(k_{2}\right) \\
& \text { add } \rightarrow \text { products }\left(k_{3}\right)
\end{aligned}
$$

Any decomposition of the adduct to products other than $\mathrm{OH}$ will also increase $k_{3}$. Moreover, $k_{3}$ may be increased deliberately by addition of reactants such as $\mathrm{O}_{2}, \mathrm{NO}$, or $\mathrm{NO}_{2}$ to study the kinetics of the corresponding adduct reactions. ${ }^{6,7}$

The analytical solution of the system of differential equations corresponding to reactions (R1a/-1a), (R1b), (R2), and (R3) leads to biexponential decay curves for $\mathrm{OH}^{4}{ }^{4}$

$$
\begin{gathered}
{[\mathrm{OH}]=C_{1} \exp \left(-t / \tau_{1}\right)+C_{2} \exp \left(-t / \tau_{2}\right)} \\
\tau_{1,2}^{-1}=\frac{a+d}{2} \pm \sqrt{\left(\frac{a-d}{2}\right)^{2}+b c}
\end{gathered}
$$

The coefficients $a, b, c$ and $d$ are related to the rate constants by the following equations:

$$
\begin{gathered}
a=\left(k_{1 \mathrm{a}}+k_{1 \mathrm{~b}}\right) \text { [aromatic] }+k_{2} \\
b=k_{-1 \mathrm{a}}
\end{gathered}
$$

$$
\begin{gathered}
c=k_{1 \mathrm{a}} \text { [aromatic] } \\
d=k_{-1 \mathrm{a}}+k_{3}
\end{gathered}
$$

Moreover, under experimental conditions with $[\operatorname{add}]_{0}=0$, i.e. pulsed formation of $\mathrm{OH}$ at $t=0$, the following expression can be derived for the ratio of the amplitudes $C_{1}$ and $C_{2}$ of the two exponentials at $t=0$ (Section $\mathrm{S} 1, \mathrm{ESI} \dagger)$ :

$$
C_{1 / 2}=C_{1} / C_{2}=\frac{\tau_{1}^{-1}-d}{d-\tau_{2}^{-1}}
$$

Under pseudo-first-order conditions, i.e. independent of the $\mathrm{OH}$ starting concentration, a biexponential $\mathrm{OH}$ decay curve is therefore described by three curve parameters: $C_{1 / 2}, \tau_{1}^{-1}$ and $\tau_{2}^{-1}$. These curve parameters can be calculated from the three coefficients $a, d$ and the product $b c$ related to the rate constants of the reactions involved, and vice versa. However, the product $b c$ cannot be separated, i.e. the system is generally under-determined. A more general solution also covering the case where $[\mathrm{add}]_{0} \neq 0$ is given in the $\mathrm{ESI} \dagger$ (Section $\mathrm{S} 1$ ).

3.1.2 Formation of two adduct species - model-2. With two adducts the chemical reaction scheme can be described as follows:

$$
\begin{array}{ll}
\mathrm{OH}+\operatorname{aromatic} \rightleftharpoons \operatorname{add}_{1}\left(k_{11 \mathrm{a}}, k_{-11 \mathrm{a}}\right) & (\mathrm{R} 11 \mathrm{a} /-11 \mathrm{a}) \\
\mathrm{OH}+\operatorname{aromatic} \rightleftharpoons \operatorname{add}_{2}\left(k_{12 \mathrm{a}}, k_{-12 \mathrm{a}}\right) & (\mathrm{R} 12 \mathrm{a} /-12 \mathrm{a})
\end{array}
$$

Irreversible losses of $\mathrm{OH}$ and the adducts are treated similarly as in the case of one adduct, i.e. by reactions (R1b) and (R2), and the following two reactions:

$$
\begin{aligned}
& \operatorname{add}_{1} \rightarrow \text { products }\left(k_{31}\right) \\
& \operatorname{add}_{2} \rightarrow \text { products }\left(k_{32}\right)
\end{aligned}
$$

Solution of the corresponding system of differential equations now yields triexponential decay curves for $\mathrm{OH}$ :

$$
[\mathrm{OH}]=C_{1} \exp \left(-t / \tau_{1}\right)+C_{2} \exp \left(-t / \tau_{2}\right)+C_{3} \exp \left(-t / \tau_{3}\right)
$$

The expressions for the decay rate coefficients $\tau_{1-3}^{-1}$ are more complicated, but can basically be expressed as a function of three parameters $r, s$, and $u$ (Section $\mathrm{S} 2, \mathrm{ESI} \dagger)$ :

$$
\begin{gathered}
r=-a-d-g \\
s=a d+d g+a g-b c-e f \\
u=b c g+e f d-a d g
\end{gathered}
$$

The coefficients $a, b, c, d, e$ and $f$ are again related to the rate constants:

$$
\begin{gathered}
a=\left(k_{11 \mathrm{a}}+k_{12 \mathrm{a}}+k_{1 \mathrm{~b}}\right)[\text { aromatic }]+k_{2} \\
b=k_{-11 \mathrm{a}} \\
c=k_{11 \mathrm{a}}[\text { aromatic] } \\
d=k_{-11 \mathrm{a}}+k_{31} \\
e=k_{-12 \mathrm{a}} \\
f=k_{12 \mathrm{a}}[\text { aromatic }] \\
g=k_{-12 \mathrm{a}}+k_{32}
\end{gathered}
$$


With $\left[\operatorname{add}_{1}\right]_{0}=0$ and $\left[\operatorname{add}_{2}\right]_{0}=0$, equations were derived for the ratios of the initial values $C_{1 / 2}$ and $C_{3 / 2}$ (Section $\mathrm{S} 2, \mathrm{ESI} \dagger$ ).

$$
\begin{aligned}
& C_{1 / 2}=C_{1} / C_{2}=\frac{\left(d-\tau_{1}^{-1}\right)\left(g-\tau_{1}^{-1}\right)\left(\tau_{3}^{-1}-\tau_{2}^{-1}\right)}{\left(d-\tau_{2}^{-1}\right)\left(g-\tau_{2}^{-1}\right)\left(\tau_{1}^{-1}-\tau_{3}^{-1}\right)} \\
& C_{3 / 2}=C_{3} / C_{2}=\frac{\left(d-\tau_{3}^{-1}\right)\left(g-\tau_{3}^{-1}\right)\left(\tau_{2}^{-1}-\tau_{1}^{-1}\right)}{\left(d-\tau_{2}^{-1}\right)\left(g-\tau_{2}^{-1}\right)\left(\tau_{1}^{-1}-\tau_{3}^{-1}\right)}
\end{aligned}
$$

Triexponential $\mathrm{OH}$ decay curves are therefore characterized by five curve parameters: $C_{1 / 2}, C_{3 / 2}, \tau_{1}^{-1}, \tau_{2}^{-1}$ and $\tau_{3}^{-1}$. These curve parameters can be calculated from the five coefficients $a, d, g$, and the products $b c$ and $e f$ related to the rate constants of the reactions involved, and vice versa. The products $b c$ and ef cannot be separated, i.e. the system is again under-determined. Moreover, as is evident from eqn (19) and (20), the variables $d$ and $g$ are exchangeable. Thus we arbitrarily chose $d>g$ to distinguish between $\operatorname{add}_{1}$ and $\operatorname{add}_{2}$. In the ESI $\dagger$ (Section S2) we give more general expressions for the case $\left[\operatorname{add}_{1}\right]_{0} \neq 0$ and/or $\left[\operatorname{add}_{2}\right]_{0} \neq 0$ which may be useful for other applications. To our knowledge these analytical solutions have not been published before. Preliminary results based on this reaction model were presented during a Workshop on Atmospheric Chemistry, University of Bayreuth, 24-26 February, 2010.

3.1.3 Formation of two adduct species with isomerization model-3. The possibility that has not been considered so far is that the adduct isomers convert into each other by an isomerization reaction:

$$
\operatorname{add}_{1} \rightleftharpoons \operatorname{add}_{2}\left(k_{12}, k_{21}\right)
$$

Of course that further complicates the analytical solution, but the $\mathrm{OH}$ decay curves remain triexponential and only the parameters $s$ and $u$ in eqn (10) and (11) change:

$$
\begin{gathered}
s=a d+d g+a g-b c-e f-h i \\
u=b c g+e f d-a d g+e i c+h i a+f b h
\end{gathered}
$$

The new coefficients $i$ and $h$ are related to the isomerization reaction rate constants:

$$
i=k_{12} \quad \text { and } \quad h=k_{21}
$$

Obviously the product $h i$ is a further fit parameter that has to be determined here. In addition the "mixed" products eic and $f b h$ seem to complicate things further. However, the rate constants involved in this extended mechanism were assumed to obey an additional relation that comes from detailed balancing considerations: ${ }^{21}$

$$
\frac{k_{12 \mathrm{a}}}{k_{-12 \mathrm{a}}} \frac{k_{21}}{k_{12}} \frac{k_{-11 \mathrm{a}}}{k_{11 \mathrm{a}}}=1
$$

In terms of the above parameters that means:

$$
f b h=e i c=\sqrt{b c e f h i}
$$

Setting $\left[\operatorname{add}_{1}\right]_{0}=0$ and $\left[\operatorname{add}_{2}\right]_{0}=0$ ratios of initial values $C_{1 / 2}$ and $C_{3 / 2}$ were again obtained (Section S3, ESI $\dagger$ ). Also the triexponential $\mathrm{OH}$ decay curves of model-3 are described by five curve parameters: $C_{1 / 2}, C_{3 / 2}, \tau_{1}^{-1}, \tau_{2}^{-1}$ and $\tau_{3}^{-1}$ but in this case they have to be calculated from six coefficients: $a, d, g$, and the products $b c$, ef and $h i$ related to the reaction rate constants.
The products $b c$, ef and $h i$ cannot be separated, as before. Moreover, the fact that six coefficients determine five curve parameters already implies that there is no unique relationship between these quantities.

\subsection{Data analysis}

The simplest approach to evaluate $\mathrm{OH}$ decay curves is to fit the curve parameters for each curve separately using a nonlinear least-squares fitting procedure. For a biexponential decay these parameters are $C_{1 / 2}, \tau_{1}^{-1}$ and $\tau_{2}^{-1}$. For a triexponential decay $C_{1 / 2}, C_{3 / 2}, \tau_{1}^{-1}, \tau_{2}^{-1}$, and $\tau_{3}^{-1}$ must be determined. Additional parameters to fully describe an experimental curve are the starting signal, i.e. a count rate proportional to $[\mathrm{OH}]_{0}$, and the background signal. That gives a total of five or seven fit parameters for each curve, dependent on the chemical model.

Taking a biexponential decay curve as an example, the three fitted curve parameters can be converted to the coefficients $a, d$ and $b c$ (Section $\mathrm{S} 1, \mathrm{ESI} \dagger$ ). $\mathrm{OH}$ decay curves were measured at various aromatics concentrations. Plainly, a linear regression of $a$ as a function of [aromatic] would then give a slope and an intercept corresponding to the sum of rate constants $k_{1 \mathrm{a}}+k_{1 \mathrm{~b}}$ and $k_{2}$, respectively (eqn (3)). The linearity of the dependence of $a$ on [aromatic] as well as the constancy of $d$ for a given temperature can serve as a test for the validity of the kinetic model. Moreover, to isolate $b$ and $c$, it can be assumed that for example in the case of benzene $k_{1 \mathrm{~b}}=0$ as a good approximation, i.e. $c=a-k_{2}$ (no reaction except addition). For methyl-substituted aromatics $k_{1 \mathrm{~b}}$ can be estimated from an extrapolation of high-temperature abstraction rate constants, ${ }^{22}$ i.e. $c=a-k_{1 \mathrm{~b}}$ [aromatic] $-k_{2}$ (eqn (3) and (5)).

However, fitting curve parameters of each decay curve separately is not the best method of data analysis. The range of useful experimental conditions can be extended by an approach where several decay curves obtained at different reactant concentrations are fitted simultaneously. The advantage is that also curves where a single fit would be critical because the reciprocal lifetimes approach each other or the amplitude ratio $C_{1 / 2}$ is getting too small or too great, are still useful if evaluated together with other curves. Moreover, the procedures described above consist of two steps to finally derive rate constants. On the other hand, the rate constants can be obtained directly as parameters from a simultaneous fit to all decay curves measured at constant temperature and pressure. In these fits the experimental errors of all data points and their influence on the rate constants are taken into account more directly and consistently. This approach was used successfully and was described in previous work ${ }^{6,7}$ but was so far confined to the reaction model resulting in biexponential decay curves.

In the present study isothermal arrays of decay curves were fitted simultaneously using the programming language IDL by Research Systems Inc. For model-1 (biexponential curves), a fit-function was defined that - upon input of $m$ experimental reactant concentrations, and arrays of measurement times and interval widths - calculates the $m$ decay curves from $4+2 m$ fit parameters (Section S4, ESI $\dagger$ ). The parameters are (1) $k_{2},(2)$ $k_{1 \mathrm{a}}+k_{1 \mathrm{~b}}=\left(a-k_{2}\right) /\left[\right.$ aromatic], (3) $k_{1 \mathrm{a}} k_{-1 \mathrm{a}}=b c /$ [aromatic], and (4) $k_{-1 \mathrm{a}}+k_{3}=d$, as well as the $m$ initial count rates $S_{0}$ 
and backgrounds $S_{\mathrm{B}}$ for each curve. Reasonable starting values for $S_{0}$ and $S_{\mathrm{B}}$ were obtained from pre-fits to the individual curves. From the starting values of the first four parameters and the aromatics concentrations the coefficients $a$, $b c, d$ and the resulting $C_{1 / 2}$ and $\tau_{1,2}^{-1}$ were calculated for each curve. Optimization of all fit parameters by comparison with the experimental curves was then accomplished by a nonlinear Levenberg-Marquardt fitting procedure. ${ }^{23}$ In the fits each data point was weighted according to Poisson counting statistics, i.e. with a ratio $n / N$ where $n$ is the number of originally accumulated decays and $N$ is the number of counted photons (Section S4, ESI $\dagger$ ).

The procedure to fit arrays of triexponential decay curves was technically similar but the number of optimized parameters was greater: $6+2 m$. The first six parameters for model-2 are (1) $k_{2}$, (2) $k_{11 \mathrm{a}}+k_{12 \mathrm{a}}+k_{1 \mathrm{~b}}=\left(a-k_{2}\right) /$ [aromatic], (3) $k_{11 \mathrm{a}} k_{-11 \mathrm{a}}=b c /\left[\right.$ aromatic], (4) $k_{12 \mathrm{a}} k_{-12 \mathrm{a}}=e f /$ [aromatic], (5) $k_{-11 \mathrm{a}}+k_{31}=d$, and (6) $k_{-12 \mathrm{a}}+k_{32}=g$. For model-3 we also fitted six parameters: (1) $k_{2}$, (2) $k_{11 \mathrm{a}}+k_{12 \mathrm{a}}+$ $k_{1 \mathrm{~b}}=\left(a-k_{2}\right) /\left[\right.$ aromatic], (3) $k_{11 \mathrm{a}} k_{-11 \mathrm{a}}=b c /$ [aromatic], (4) $k_{12} k_{21}=h i,(5) k_{-11 \mathrm{a}}+k_{12}+k_{31}=d$, and (6) $k_{21}+k_{32}=g$. The parameter $e f /[$ aromatic] was held fixed at a very small value, i.e. $k_{12 \mathrm{a}} k_{-12 \mathrm{a}} \approx 0$, to simulate a case of $\mathrm{add}_{2}$ formation only by isomerization. A full fit adjusting all seven parameters of model-3 was not performed as will be explained below.

No attempt was made to individually analyze single decay curves, neither biexponential, nor triexponential because there is no reason for such a step backward. Considering groups of decay curves obtained at the same temperature is the optimum procedure to identify and quantify any differences between the various model approaches. Whether or not the applied chemical models are consistent with the experimental data can be assessed from the fitted sum of weighted squared residuals $\chi^{2}$ divided by the degrees of freedom. The degrees of freedom (DOF) are the number of data points minus the number of fitted parameters, i.e. $59 m-4$ for a model-1 fit and $59 m-6$ for a model-2 or model-3 fit, respectively. Deviations of $\chi^{2} / \mathrm{DOF}$ towards values much greater than unity indicate that the applied fit function is not suitable or that experimental errors were underestimated. On the other hand, values well below unity in any case indicate an overestimation of experimental errors. Thus a thorough assessment of experimental errors in the fitting procedure is crucial for the judgement of fit quality and the applicability of a fit function. Based on simulated experimental data for model-1 and model-2 including experimental random noise according to Poisson statistics we confirmed that the fit routines on average return $\chi^{2} / \mathrm{DOF}=$ 1.00 with a standard deviation of about 0.08 (Section S5.1, ESI $\dagger$ ). For experimental data somewhat greater values are expected because a mean value $\chi^{2} / \mathrm{DOF}=1.00$ is a theoretical limit in the case of accurately known experimental errors and data that without these errors are in perfect agreement with the underlying model (as in the simulations). Typically there are additional, unaccounted sources of errors that lead to somewhat greater values.

Error limits of the fitted parameters were estimated as recently introduced in similar applications of the least-squares fitting procedure. ${ }^{24}$ Successively, the four (model-1) and six (model-2, model-3) parameters were stepwise increased or decreased starting with the optimum values and held fixed in the fits until the ratio $\chi^{2} / \mathrm{DOF}$ increased by a predefined factor. This factor ranged between 1.02 and 1.03 dependent on DOF and was taken from a parametrization of the $\chi^{2}$-distribution for a probability of 0.68 . All other parameters were allowed to adjust freely during these fits, i.e. the resulting ranges reflect error limits that cover the mutual dependence of the fit parameters. The original idea was that this procedure results in estimated $1 \sigma$ error limits. However, based on the simulated experimental data it turned out that these errors are greater by about a factor of three compared to the standard deviations of the parameters resulting from the simulations (Section S5.3, ESI $\dagger$ ). The error estimates are therefore rather conservative but nevertheless they cannot account for systematic deviations that arise when the applied chemical model is incorrect.

\subsection{Comparison of model-1 and model-2 results}

Fig. 1 shows examples of normalized $\mathrm{OH}$ decay curves obtained in the presence of various 1,3,5-TMB concentrations at $324 \mathrm{~K}$ (experiment 10, Table 1). Only four curves (out of the total array of seven that were fitted simultaneously) are shown for clarity. As mentioned before, actual measurement times extended up to $5 \mathrm{~s}$ for an accurate determination of the background that was subtracted in the displayed plots but considered in the error bars. The unequal spacing of the data points is caused by the data compression. The full lines are fits according to model-1 and model-2. Because fitted background levels and starting values were slightly different for the two models, also the data points in Fig. 1 differ slightly. In this example, the triexponential model-2 clearly describes the data better than the biexponential model-1 as will be discussed in more detail below.

Fitted parameters of model-1 and model-2 for all 1,3,5TMB experiments are listed in Tables 1 and 2 and plotted in Fig. 2 in semi-logarithmic representations as a function of reciprocal temperatures (Arrhenius plots). In addition, $\chi^{2} / \mathrm{DOF}$ is shown on a linear scale. For experiments $1-3$ at temperatures below $290 \mathrm{~K}$, fits using model-2 either failed to converge or became biexponential, i.e. identical to model-1. However, towards higher temperatures the two models led to significantly different results. Moreover, while the quality of the fits of model-1 decreased with rising temperature, that of model-2 remained in an acceptable range with $\chi^{2} / \mathrm{DOF} \leq$ 1.5. This is clear evidence that model-2 is in better accordance with the experimental data than model-1, at least at higher temperatures. Obviously two species with significantly different properties are formed in the reaction of $\mathrm{OH}$ with 1,3,5-TMB. Although no structural information was obtained here, we assume that these species are ortho- and ipso-type adducts.

Independent of the applied model, no dependence on total pressure was found at 380 or $750 \mathrm{hPa}$ of $\mathrm{He}$. Thus, all reactions were at their high pressure limits - in accordance with previous work also on other aromatics. ${ }^{6,25,26}$ The $\mathrm{OH}$ background loss rate constant $k_{2}$ decreased significantly with increasing temperature and showed little dependence on total pressure. This behaviour was confirmed by measurements in the absence of added aromatics. The nature of the background 

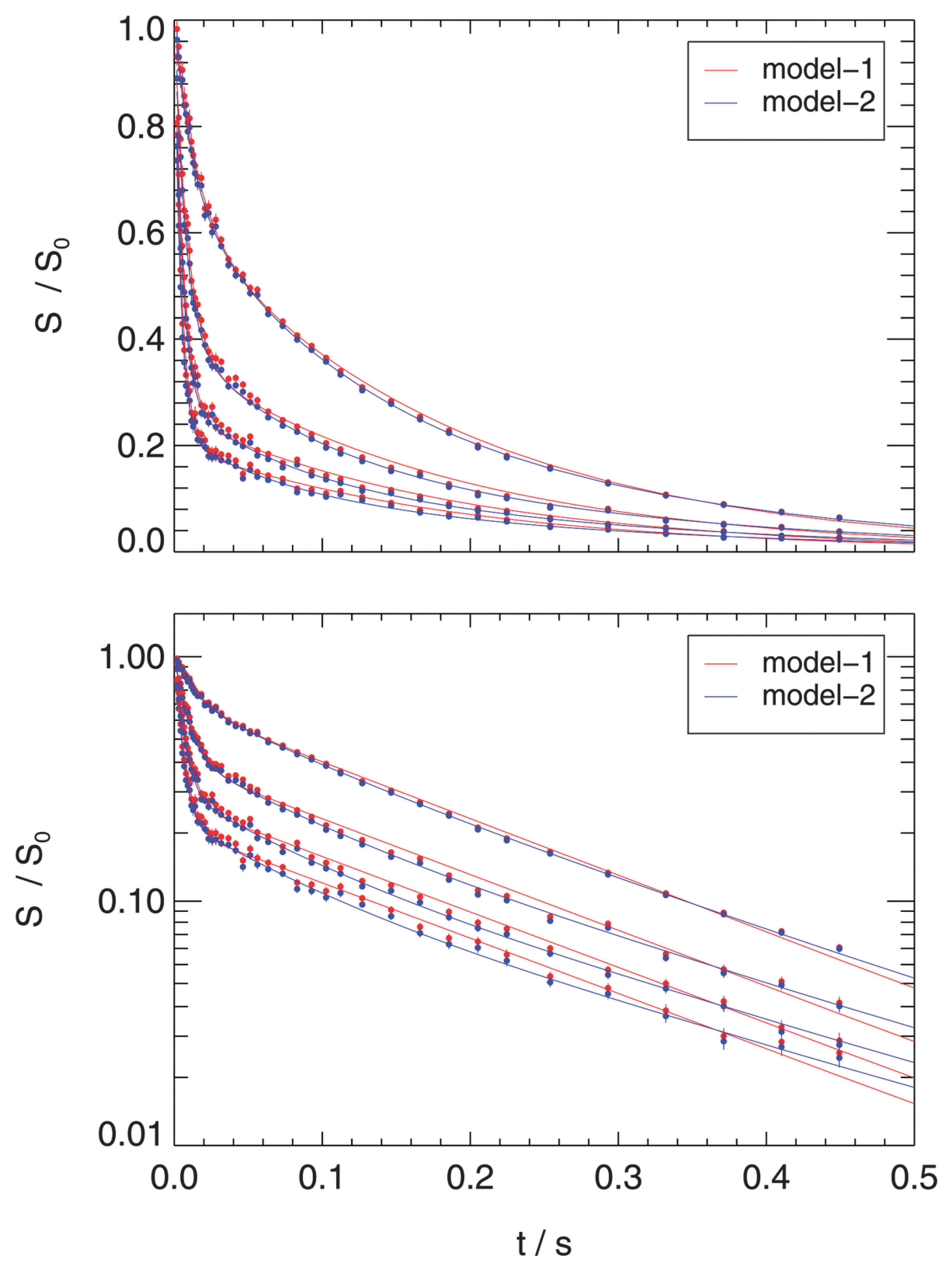

Fig. 1 Examples of $\mathrm{OH}$ decay curves in linear and semi-logarithmic representations. Four from a total of seven decay curves of experiment 10 with 1,3,5TMB at $324 \mathrm{~K}$ are shown. 1,3,5-TMB concentrations increase from top to bottom (in units $10^{12} \mathrm{~cm}^{-3}: 0.48,1.42,2.39$, 3.31). Full lines are fits to all curves simultaneously using model-1 (red) and model-2 (blue). Data points were calculated from photon counts $N$ divided by interval widths and assigned to the middle of the interval. Fitted background levels were subtracted and the data were then normalized by the fitted starting count rates for better comparability.

loss is unknown but is probably dominated by impurities because diffusional losses would increase at decreasing pressure and increasing temperature. However, the influence of $k_{2}$ on the other results is minor and the fitted values were very similar for both models.

Based on numerical simulations we confirmed that neither radical-radical reactions nor diffusion processes can lead to deviations that would pretend a different reaction model (Sections S5.1 and S5.2, ESI $\dagger$ ). The simulation results are supported by a series of experiments with 1,2,4-TMB where the flash-energy was lowered by a factor of two with no noticeable effect on fitted rate constants (Section S6, ESI $\dagger$ ) and by the independence of total pressure.
3.3.1 OH + TMB rate constants. The second-order rate constants $k_{\mathrm{OH}}$ of $\mathrm{OH}+1,3,5-\mathrm{TMB}$ were obtained directly as fit parameters and assigned to the sum $k_{1 \mathrm{a}}+k_{1 \mathrm{~b}}$ for model- 1 and $k_{11 \mathrm{a}}+k_{12 \mathrm{a}}+k_{1 \mathrm{~b}}$ for model-2, respectively. The rate constants obtained here for model-1 are identical to those determined previously ${ }_{10}^{10}$ confirming that the different software tools worked consistently. At temperatures below about $300 \mathrm{~K}$ both models gave the same rate constants. Moreover, for both models $k_{\mathrm{OH}}$ decreased with increasing temperature. However, towards higher temperatures the rate constants of model-1 show a stronger decrease. Because of the poorer fit quality we consider this strong decrease as an artefact of the data analysis of model- 1 while the results of model-2 are presumed to be correct. 
Table 2 Triexponential model-2 fit results and estimated error limits from simultaneous fits to $m$ decay curves at different $1,3,5$-TMB concentrations (see Table 1 for $m$ and experimental conditions)

\begin{tabular}{|c|c|c|c|c|c|c|c|c|}
\hline \# & $k_{2} / \mathrm{s}^{-1}$ & $\begin{array}{l}k_{11 \mathrm{a}}+k_{12 \mathrm{a}}+k_{1 \mathrm{~b}} / \\
10^{-11} \mathrm{~cm}^{3} \mathrm{~s}^{-1}\end{array}$ & $\begin{array}{l}k_{11 \mathrm{a}} k_{-11 \mathrm{a} /} \\
10^{-10} \mathrm{~cm}^{3} \mathrm{~s}^{-2}\end{array}$ & $\begin{array}{l}k_{12 \mathrm{a}} k_{-12 \mathrm{a}} / \\
10^{-10} \mathrm{~cm}^{3} \mathrm{~s}^{-2}\end{array}$ & $k_{-11 \mathrm{a}}+k_{31} / \mathrm{s}^{-1}$ & $k_{-12 \mathrm{a}}+k_{32} / \mathrm{s}^{-1}$ & DOF & $\chi^{2} / \mathrm{DOF}$ \\
\hline 4 & $9.7 \pm 3.9$ & $6.22 \pm{ }_{0.30}^{0.32}$ & $1.85 \pm{ }_{0.79}^{0.73}$ & $0.67 \pm{ }_{0.48}^{0.61}$ & $15.9 \pm 7.1$ & $3.3 \pm{ }_{2.0}^{1.8}$ & 348 & 0.95 \\
\hline 5 & $14.7 \pm 2.5$ & $5.91 \pm 0.24$ & $2.35 \pm 0.50$ & $0.83 \pm{ }_{0.57}^{0.77}$ & $12.3 \pm 8.7$ & $3.7 \pm 1.3$ & 407 & 1.32 \\
\hline 6 & $9.8 \pm 0.9$ & $5.98 \pm 0.18$ & $2.67 \pm 0.54$ & $0.82 \pm \begin{array}{l}0.59 \\
0.48\end{array}$ & $14.1 \pm \begin{array}{l}6.8 \\
3.7\end{array}$ & $4.0 \pm 1.1$ & 407 & 1.10 \\
\hline 7 & $10.1 \pm 1.5$ & $5.82 \pm 0.21$ & $4.52 \pm 0.77$ & $0.62 \pm \begin{array}{l}0.90 \\
0.41\end{array}$ & $14.7 \pm 2.6$ & $4.5 \pm 1.9$ & 407 & 1.28 \\
\hline 8 & $8.8 \pm 0.6$ & $5.46 \pm \begin{array}{l}0.22 \\
0.19\end{array}$ & $10.7 \pm 0.83$ & $0.47 \pm{ }_{0.23}^{0.43}$ & $27.3 \pm 2.0$ & $6.4 \pm \begin{array}{l}1.9 \\
1.6\end{array}$ & 407 & 1.27 \\
\hline 9 & $7.8 \pm 0.4$ & $5.71 \pm{ }_{0.31}^{0.35}$ & $17.9 \pm 2.1$ & $0.32 \pm \begin{array}{l}0.39 \\
0.17\end{array}$ & $39.6 \pm \frac{4.2}{3.2}$ & $7.0 \pm \begin{array}{l}3.0 \\
2.1\end{array}$ & 407 & 1.57 \\
\hline 10 & $5.4 \pm 0.3$ & $5.46 \pm \begin{array}{l}0.51 \\
0.45\end{array}$ & $27.0 \pm 3.7$ & $0.33 \pm \begin{array}{l}0.41 \\
0.18\end{array}$ & $59.6 \pm \begin{array}{l}7.3 \\
5.7\end{array}$ & $9.0 \pm 2.8$ & 407 & 1.31 \\
\hline 11 & $4.9 \pm 0.2$ & $5.00 \pm 0.89$ & $54.9 \pm 12.8$ & $0.26 \pm \begin{array}{l}0.32 \\
0.14\end{array}$ & $125 \pm \begin{array}{l}17 \\
14\end{array}$ & $13.2 \pm 5.1$ & 407 & 1.38 \\
\hline 12 & $4.9 \pm 0.2$ & $4.81 \pm 0.98$ & $50.0 \pm 12.8$ & $0.12 \pm 0.60$ & $117 \pm \frac{23}{15}$ & $10.8 \pm \begin{array}{l}13 \\
6.5\end{array}$ & 407 & 1.27 \\
\hline 13 & $5.1 \pm 0.1$ & $5.43 \pm 1.07$ & $99.2 \pm \begin{array}{l}36.0 \\
24.7\end{array}$ & $0.38 \pm \begin{array}{l}0.58 \\
0.24\end{array}$ & $205 \pm 24$ & $23.1 \pm 8.0$ & 407 & 1.54 \\
\hline 14 & $5.1 \pm 0.2$ & $5.37 \pm 1.35$ & $107 \pm 33$ & $0.18 \pm 0.09$ & $221 \pm{ }_{29}^{34}$ & $14.3 \pm 4.6$ & 407 & 1.79 \\
\hline
\end{tabular}

In contrast to those of model-1, the rate constants of model-2 can be described empirically by a straight Arrhenius dependence $k_{\mathrm{OH}}=A_{1} \exp \left(-B_{1} / T\right)$ as indicated by the full blue line in the upper panel of Fig. 2. Only a minor difference was obtained using a combination of data from model-2 above $290 \mathrm{~K}$ and of model-1 below $290 \mathrm{~K}$ (dashed blue line in Fig. 2). Because the latter combination is covering a wider temperature range we prefer the corresponding Arrhenius parameters that are given in Table 3. The simpler temperature dependence resulting from model-2 is in agreement with literature data by Aschmann et $a .^{27}$ obtained with a relative rate method in a similar range of temperatures as indicated by the dashed black line in Fig. 2. Although the absolute values are somewhat smaller compared with the results of this work, the general temperature dependence is similar and confirms the advantage of model-2. Table 3 also lists other room temperature rate constants of $\mathrm{OH}+1,3,5-$ TMB from the literature that are in good agreement with that of this work.

The fit results obtained with 1,2,3-TMB and 1,2,4-TMB will not be discussed here in detail because the model-2 approach does not strictly apply to these compounds. Nevertheless, by switching from model-1 to model-2 we obtained qualitatively similar results, namely a significant improvement of fit qualities towards greater temperatures and a weaker decrease of $k_{\mathrm{OH}}$ with temperature. Fit results and plots can be found in the $\mathrm{ESI} \dagger$ (Section S6). The improvement of the fit quality can be rationalized by the fact that the four and six possible isomers for 1,2,3-TMB and 1,2,4-TMB fall into two groups of orthoand ipso-type adducts with presumably similar properties within the groups. Consequently, we assume that also the $k_{\mathrm{OH}}$ are more reliable than those obtained with model-1 that were published previously. ${ }^{10}$ As for $1,3,5-\mathrm{TMB}$, the $k_{\mathrm{OH}}$ can now be described by straight Arrhenius expressions in good approximation. The corresponding parameters, together with a comparison of available room temperature rate constants from the literature are given in Table 3 .

3.3.2 Adduct loss rate constants. In contrast to the $k_{\mathrm{OH}}$, the fitted first-order rate constants of adduct loss of 1,3,5-TMB, $k_{\text {add }}$, increased with temperature and levelled out to small values at low temperatures as shown in the second panel of Fig. 2. These rate constants correspond to the sums $k_{-1 \mathrm{a}}+k_{3}$ for model-1, as well as $k_{-11 \mathrm{a}}+k_{31}\left(\operatorname{add}_{1}\right)$ and $k_{-12 \mathrm{a}}+k_{32}\left(\operatorname{add}_{2}\right)$ for model-2. In fact, with model-2 two strongly different $k_{\text {add }}$ were obtained while that for model-1 expectedly lies in between these extremes, albeit at the expense of a poorer fit quality. Again we conclude that model-2 may describe the actual properties of two adduct isomers while the result of model-1 is an inadequate compromise. The full lines in Fig. 2 show that the temperature dependencies of all three quantities can be described by modified Arrhenius expressions $k_{\text {add }}=A_{2}$ $\exp \left(-B_{2} / T\right)+C$ that allowed for a constant, temperature independent contribution of background loss reactions. Unlike the $k_{2}$, that could be determined independently and separated from $k_{\mathrm{OH}}$, the adduct background loss cannot be measured directly. Therefore we assumed temperature independent contributions $(C)$ of $k_{3}, k_{31}$ and $k_{32}$ while the $k_{-1 \mathrm{a}}, k_{-11 \mathrm{a}}$ and $k_{-12 \mathrm{a}}$ were found to increase exponentially with temperature. The three different $A_{2}, B_{2}$ and $C$ are listed in Table 4 . The $A_{2}$ vary over many orders of magnitude. Because of the narrow range of temperatures investigated here, this parameter is extremely uncertain. On the other hand, the $B_{2}$ and $C$ are comparable and lie in a range $5000-10000 \mathrm{~K}$ and $1-8 s^{-1}$, respectively. Uncertainties for these parameters were estimated by fitting maximum and minimum values of the data in Tables 1 and 2. Within these limits the $B_{2}$ of the two adduct isomers of model-2 are significantly different but unexpectedly the smaller $k_{\text {add }}$ of $\operatorname{add}_{2}$ corresponds to a smaller $B_{2}$ because of the extremely small $A_{2}$. Despite the uncertainties caused by the narrow $T$-range, the $A_{2}$ of $\operatorname{add}_{2}$ seems unrealistically low because preexponential factors usually range around $10^{11}-10^{16} \mathrm{~s}^{-1}$ for unimolecular reactions. ${ }^{28}$

Previous studies on benzene, ${ }^{6,13}$ toluene ${ }^{6,13}$ and $p$-xylene ${ }^{14}$ gave very similar results for $B_{2}$ and $C$ in a range $7700-8800 \mathrm{~K}$ and $2.5-5.5 \mathrm{~s}^{-1}$, respectively (Table 4 ). This matches very well with the result obtained here for 1,3,5-TMB with model-1 and is also in reasonable agreement with the properties of $\operatorname{add}_{1}$ of model-2. For example, taking a typical experimental temperature of $320 \mathrm{~K}$, the values for $k_{\text {add }}$ range between 20 and $30 \mathrm{~s}^{-1}$ for benzene, toluene, $p$-xylene and 1,3,5-TMB, all based on model-1. For model-2 this rate constant only slightly increases to $36 \mathrm{~s}^{-1}$ for $\operatorname{add}_{1}$ but drops more strongly to $6 \mathrm{~s}^{-1}$ for $\operatorname{add}_{2}$. Because a single, high-temperature study on hexamethylbenzene $(\mathrm{HMB})^{29}$ revealed the existence of a very stable ipso-adduct with an extrapolated dissociation rate constant of $0.6 \mathrm{~s}^{-1}$ at $320 \mathrm{~K}$, we therefore tentatively identify $\operatorname{add}_{2}$ as the ipso-isomer and $\operatorname{add}_{1}$ 

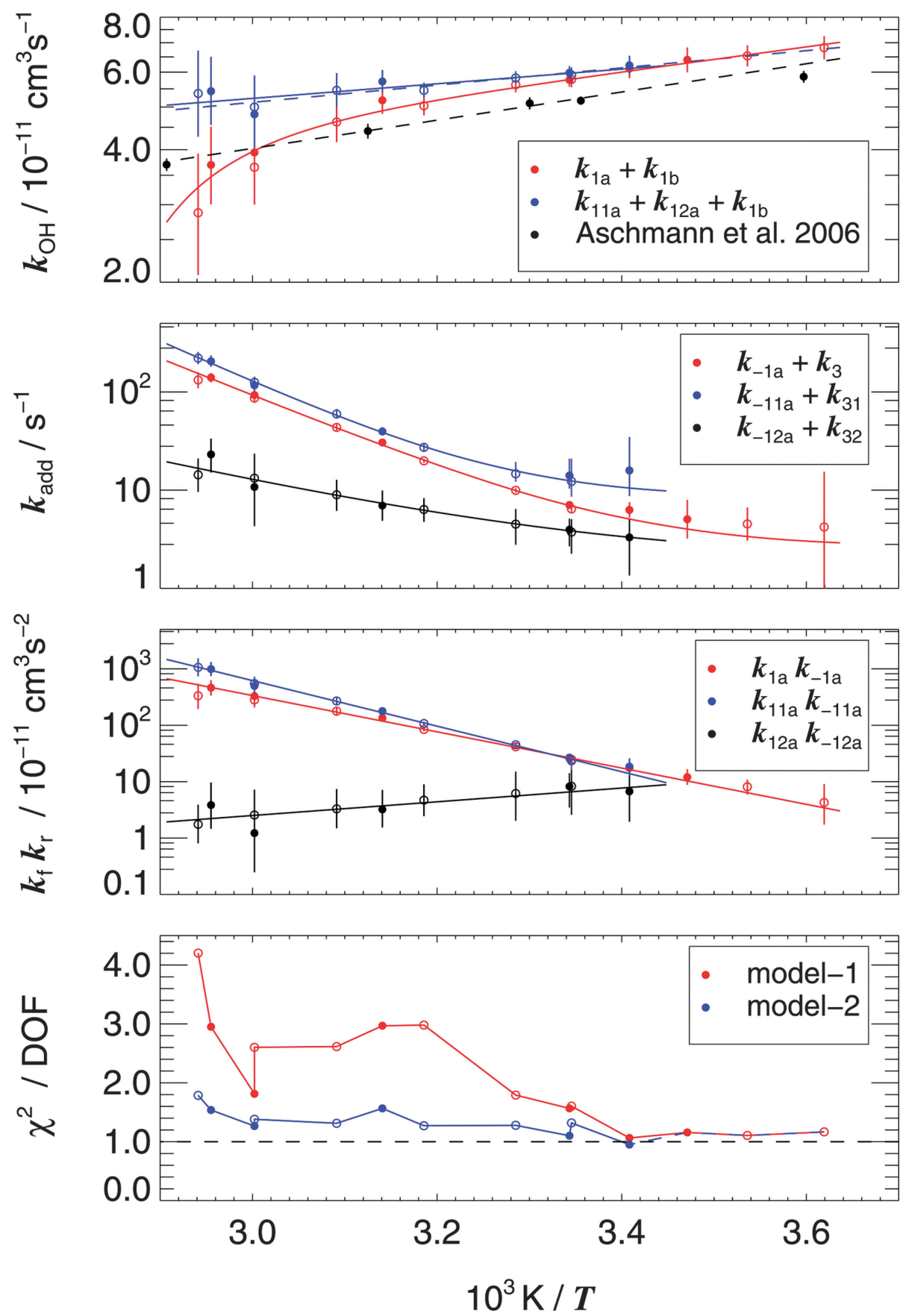

Fig. 2 Rate constant related fit parameters and fit qualities $\chi^{2}$ /DOF for 1,3,5-TMB using model-1 (red) and model-2 (black, blue). Open symbols refer to measurements at $380 \mathrm{mbar}$ and filled symbols at $750 \mathrm{mbar}$. Full lines in the upper three panels correspond to fitted Arrhenius expressions except for the red line in the first panel that shows a previous parametrization from the literature. ${ }^{10}$ The dashed blue line in the first panel is an Arrhenius fit using model-2 data above $290 \mathrm{~K}$ together with model-1 data below $290 \mathrm{~K}$. Black points and the dashed black line show a temperature dependence from the literature. ${ }^{27}$ In the second panel temperature independent contributions of background loss rate constants were assumed. The dashed blue lines on top of the red line in the fourth panel indicate that models converged towards low temperatures. The dashed black line shows a theoretical optimum.

as the ortho-isomer. On the other hand, the $B_{2}$ obtained for the $\mathrm{HMB}$ adduct was much greater $(10500 \mathrm{~K})^{29}$ and comparable with that of $\operatorname{add}_{1}$ of this work. Clearly more information is necessary to identify the adduct isomers.

3.3.3 Products of forward and backward rate constants, isomer yields and heats of formation. The products of the forward and reverse reactions $k_{\mathrm{f}} k_{\mathrm{r}}$ are further direct fit parameters that correspond to $k_{1 \mathrm{a}} k_{-1 \mathrm{a}}$ for model-1, as well as $k_{11 \mathrm{a}} k_{-11 \mathrm{a}}$ and $k_{12 \mathrm{a}} k_{-12 \mathrm{a}}$ for model-2. The temperature dependencies of these quantities could also be described by simple Arrhenius expressions $k_{\mathrm{f}} k_{\mathrm{r}}=A_{3} \exp \left(-B_{3} / T\right)$ in good approximation, as indicated by the full lines in the third panel of Fig. 2. The parameters $A_{3}$ and $B_{3}$ are listed in Table 5. For the $A_{3}$ the same applies as for the $A_{2}$ in the last section: these quantities are extremely uncertain because of the confined 
Table 3 Room temperature rate constants $k_{\mathrm{OH}}$ of $\mathrm{OH}+\mathrm{TMB}$ reactions and parameters $A_{1}$ and $B_{1}$ from fitted Arrhenius expressions $k_{\mathrm{OH}}=A_{1} \exp \left(-B_{1} / T\right)$ compared with literature data. The data of this work apply for a temperature range $275-340 \mathrm{~K}$ using a combination of results of model- 1 at $T<290 \mathrm{~K}$ and of model-2 at $T>290 \mathrm{~K}$ (see text). The estimated $10 \%$ systematic uncertainty of aromatics concentrations is not included in the error limits. The same applies to the results of Aschmann et al. ${ }^{27}$ where an estimated $10 \%$ uncertainty of the reference compound rate constant was not included

\begin{tabular}{|c|c|c|c|c|}
\hline Reactant & $\begin{array}{l}k_{\mathrm{OH}^{a}} / 10^{-11} \\
\mathrm{~cm}^{3} \mathrm{~s}^{-1}\end{array}$ & $\begin{array}{l}A_{1} / 10^{-12} \\
\mathrm{~cm}^{3} \mathrm{~s}^{-1}\end{array}$ & $B_{1} / 10^{3} \mathrm{~K}$ & Ref. \\
\hline \multirow[t]{7}{*}{$1,3,5-\mathrm{TMB}$} & $4.72 \pm 0.48$ & - & - & Hansen et al. ${ }^{25}$ \\
\hline & $6.24 \pm 0.75$ & - & - & Perry et al. ${ }^{26}$ \\
\hline & $5.75 \pm 0.30$ & - & - & Atkinson et al. ${ }^{38}$ \\
\hline & $5.73 \pm 0.53$ & - & - & Kramp and Paulson 39 \\
\hline & $5.91 \pm 0.11$ & - & - & Aschmann et al. ${ }^{40}$ \\
\hline & $5.17 \pm 0.11$ & 4.4 & $0.74 \pm 0.18$ & Aschmann et al. ${ }^{27}$ \\
\hline & $5.95 \pm 0.20$ & 13.2 & $\begin{array}{l}-0.45 \pm \\
0.05^{b}\end{array}$ & This work \\
\hline \multirow[t]{4}{*}{ 1,2,3-TMB } & $2.64 \pm 0.26$ & & & Hansen et al. ${ }^{25}$ \\
\hline & $3.33 \pm 0.45$ & & & Perry et al. ${ }^{26}$ \\
\hline & $3.27 \pm 0.19$ & & & Atkinson et al. . $^{38}$ \\
\hline & $2.88 \pm 0.10$ & 3.61 & $\begin{array}{l}-0.62 \pm \\
0.08^{b}\end{array}$ & This work \\
\hline \multirow[t]{4}{*}{ 1,2,4-ТMB } & $3.35 \pm 0.34$ & & & Hansen et al. ${ }^{25}$ \\
\hline & $4.00 \pm 0.45$ & & & Perry et $a l^{26}$ \\
\hline & $3.25 \pm 0.11$ & & & Atkinson et al..$^{38}$ \\
\hline & $3.05 \pm 0.20$ & 2.73 & $\begin{array}{l}-0.73 \pm \\
0.07^{b}\end{array}$ & This work \\
\hline
\end{tabular}

${ }^{a} 298 \pm 2$ K. ${ }^{b}$ Error limits from fits to maximum and minimum $k_{\mathrm{OH}}$.

temperature range. Uncertainties of $B_{3}$ were again estimated by fitting maxima and minima of the data in Tables 1 and 2 . The $k_{\mathrm{f}} k_{\mathrm{r}}$ exhibit an opposite temperature dependence for $\mathrm{add}_{1}$ and $\operatorname{add}_{2}$.

The rate constants of the forward reactions $k_{1 \mathrm{a}}, k_{11 \mathrm{a}}$ and $k_{12 \mathrm{a}}$ and thus the adduct yields can be determined from the $k_{\mathrm{f}} k_{\mathrm{r}}$ provided that the adduct background loss rate constants $k_{3}, k_{31}$ and $k_{32}$ are known. For model-1 $k_{1 \mathrm{a}}$ is given by:

$$
k_{1 \mathrm{a}}=\frac{k_{\mathrm{f}} k_{\mathrm{r}}}{k_{\mathrm{r}}}=\frac{k_{1 \mathrm{a}} k_{-1 \mathrm{a}}}{k_{-1 \mathrm{a}}}=\frac{k_{1 \mathrm{a}} k_{-1 \mathrm{a}}}{k_{\mathrm{add}}-k_{3}}=k_{\mathrm{OH}}-k_{1 \mathrm{~b}}
$$

For model-2 a similar equation applies:

$$
k_{11 \mathrm{a}}+k_{12 \mathrm{a}}=\frac{k_{11 \mathrm{a}} k_{-11 \mathrm{a}}}{k_{\mathrm{add}_{1}}-k_{31}}+\frac{k_{12 \mathrm{a}} k_{-12 \mathrm{a}}}{k_{\mathrm{add}_{2}}-k_{32}}=k_{\mathrm{OH}}-k_{1 \mathrm{~b}}
$$

Table 5 Parameters $A_{3}$ and $B_{3}$ from fitted Arrhenius expressions $k_{\mathrm{f}} k_{\mathrm{r}}=A_{3} \exp \left(-B_{3} / T\right)$ to products of forward and reverse rate constants for 1,3,5-TMB. For model-1: $k_{\mathrm{f}} k_{\mathrm{r}}=k_{1 \mathrm{a}} k_{-1 \mathrm{a}}$. For model-2: $k_{\mathrm{f}} k_{\mathrm{r}}=k_{11 \mathrm{a}} k_{-11 \mathrm{a}}\left(\operatorname{add}_{1}\right)$ and $k_{\mathrm{f}} k_{\mathrm{r}}=k_{12 \mathrm{a}} k_{-12 \mathrm{a}}\left(\operatorname{add}_{2}\right)$. For model-3: $k_{\mathrm{f}} k_{\mathrm{r}}=$ $k_{11 \mathrm{a}} k_{-11 \mathrm{a}}\left(\operatorname{add}_{1}\right), k_{\mathrm{f}} k_{\mathrm{r}}=0\left(\operatorname{add}_{2}\right)$ and $k_{\mathrm{f}} k_{\mathrm{r}}=k_{12} k_{21}\left(\operatorname{add}_{1} \rightleftharpoons \operatorname{add}_{2}\right)($ pure isomerization limit)

\begin{tabular}{llr}
\hline$k_{\mathrm{f}} k_{\mathrm{r}}$ & $A_{3} / \mathrm{cm}^{3} \mathrm{~s}^{-2}$ & \multicolumn{1}{c}{$B_{3} / 10^{3} \mathrm{~K}^{a}$} \\
\hline Mod-1 & 14.7 & $7.4 \pm 0.2$ \\
Mod-2, add & $8.1 \times 10^{3}$ & $9.3 \pm 0.2$ \\
Mod-2, $\operatorname{add}_{2}$ & $5.7 \times 10^{-15}$ & $-2.8 \pm 0.3$ \\
Mod-3, $\operatorname{add}_{1}$ & $3.3 \times 10^{2}$ & $8.3 \pm 0.2$ \\
Mod-3, $\operatorname{add}_{1} \rightleftharpoons \operatorname{add}_{2}$ & $A_{3} / \mathrm{s}^{-2}$ & \\
& $2.4 \times 10^{8}$ & $5.1 \pm 0.3$
\end{tabular}

${ }^{a}$ Error limit estimated from fits to maximum and minimum $k_{\mathrm{f}} k_{\mathrm{r}}$.

The ratios $k_{\mathrm{f}} / k_{\mathrm{OH}}$ then determine the adduct yields of the $\mathrm{OH}$ reaction.

It turned out that towards low temperatures when the denominators in eqn (27) were getting very small, the forward rate constant $k_{11 \mathrm{a}}$ became greater than $k_{\mathrm{OH}}$ which is inconsistent with the $\mathrm{OH}$ reaction balance formulated in eqn (27). Instead of the $C$ from Table 4 we therefore determined optimized values of $k_{3}, k_{31}$ and $k_{32}$ that were fitted based on eqn (26) and (27), and estimated values of $k_{1 \mathrm{~b}}$ from the literature. It was assumed that $k_{1 \mathrm{~b}}$ corresponds to the rate constant of the $\mathrm{H}$-atom abstraction reaction from the substituent $\mathrm{CH}_{3}$-groups.

Atkinson $^{22}$ derived an empirical expression for the rate constant per $\mathrm{CH}_{3}$-group from high temperature data of the $\mathrm{OH}$ reaction with toluene and xylenes ${ }^{26,30,31}$ that was extrapolated to the temperature range of this work and multiplied by three. However, these $k_{1 \mathrm{~b}}$ contribute no more than $5 \%$ to the overall $k_{\mathrm{OH}}$ and therefore have a minor influence. A similar extrapolation based on a different type of parameterisation of high temperature rate constants of toluene recommended by IUPAC $^{32}$ is leading to even smaller $k_{1 \mathrm{~b}}$ with a maximum contribution of $3 \%$ to $k_{\mathrm{OH}}$ in the temperature range considered here.

The optimized adduct background loss rate constants in terms of the $\mathrm{OH}$ reaction balance were $k_{3}=3.0 \mathrm{~s}^{-1}$, $k_{31}=1.2 \mathrm{~s}^{-1}$ and $k_{32}=1.7 \mathrm{~s}^{-1}$. Only $k_{31}$ is significantly smaller than the empirical parameter $C$ in Table 4 , but still reasonable

Table 4 Parameters $A_{2}, B_{2}$ and $C$ from fitted Arrhenius expressions $k_{\text {add }}=A_{2} \exp \left(-B_{2} / T\right)+C$ to adduct loss rate constants. For model-1: $k_{\text {add }}=k_{-1 \mathrm{a}}+k_{3}$. For model-2: $k_{\text {add }}=k_{-11 \mathrm{a}}+k_{31}\left(\operatorname{add}_{1}\right)$ and $k_{\text {add }}=k_{-12 \mathrm{a}}+k_{32}\left(\operatorname{add}_{2}\right)$. For model-3: $k_{\text {add }}=k_{-11 \mathrm{a}}+k_{12}+k_{31}\left(\operatorname{add}_{1}\right)$ and

\begin{tabular}{|c|c|c|c|c|c|}
\hline Reactant & $k_{\text {add }}$ & $A_{2} / \mathrm{s}^{-1}$ & $B_{2} / 10^{3} \mathrm{~K}$ & $C / \mathrm{s}^{-1}$ & Ref. \\
\hline \multirow[t]{5}{*}{$1,3,5-\mathrm{TMB}$} & Mod-1 & $2.5 \times 10^{13}$ & $8.8 \pm 0.7$ & $2.6 \pm 1.2$ & \multirow[t]{5}{*}{ This work $^{a}$} \\
\hline & Mod-2, $\operatorname{add}_{1}$ & $7.2 \times 10^{14}$ & $9.8+1.1$ & $8.3+5.9$ & \\
\hline & Mod-2, add $_{2}$ & $3.6 \times 10^{7}$ & $5.0 \pm 1.0$ & $1.9 \pm 1.9$ & \\
\hline & Mod-3, $\operatorname{add}_{1}$ & $3.9 \times 10^{14}$ & $9.6 \pm 0.9$ & $6.1 \pm 3.7$ & \\
\hline & Mod-3, add 2 & $3.1 \times 10^{10}$ & $7.4 \pm 3.2$ & $5.2 \pm 4.4$ & \\
\hline \multirow[t]{2}{*}{ Benzene } & Mod- $1^{b}$ & $9.0 \times 10^{12}$ & $8.6 \pm 0.2$ & $3.3 \pm 0.3$ & \multirow{6}{*}{$\begin{array}{l}\text { Knispel et al. }{ }^{6} \\
\text { Koch }^{13} \\
\text { Knispel et al. }^{6} \\
\text { Koch }^{13} \\
\text { Knispel }^{14} \\
\text { von Buttlar et al. }\end{array}$} \\
\hline & & $1.4 \times 10^{12}$ & $8.0 \pm 0.5$ & $3.0 \pm 0.3$ & \\
\hline \multirow[t]{2}{*}{ Toluene } & Mod-1 & $1.5 \times 10^{12}$ & $7.9 \pm 0.2$ & $4.8 \pm 1.8$ & \\
\hline & & $2.3 \times 10^{12}$ & $8.0 \pm 0.8$ & $5.0 \pm 0.5$ & \\
\hline$p$-Xylene & Mod-1 & $3.8 \times 10^{12}$ & $8.2 \pm 0.3$ & $4.8 \pm 1.8$ & \\
\hline Hexamethyl- & Mod-1 $1^{b}$ & $1.0 \times 10^{14}$ & 10.5 & 4.0 & \\
\hline
\end{tabular}
$k_{\text {add }}=k_{21}+k_{32}\left(\operatorname{add}_{2}\right)$ (pure isomerization limit). Literature data were obtained based on model-1 

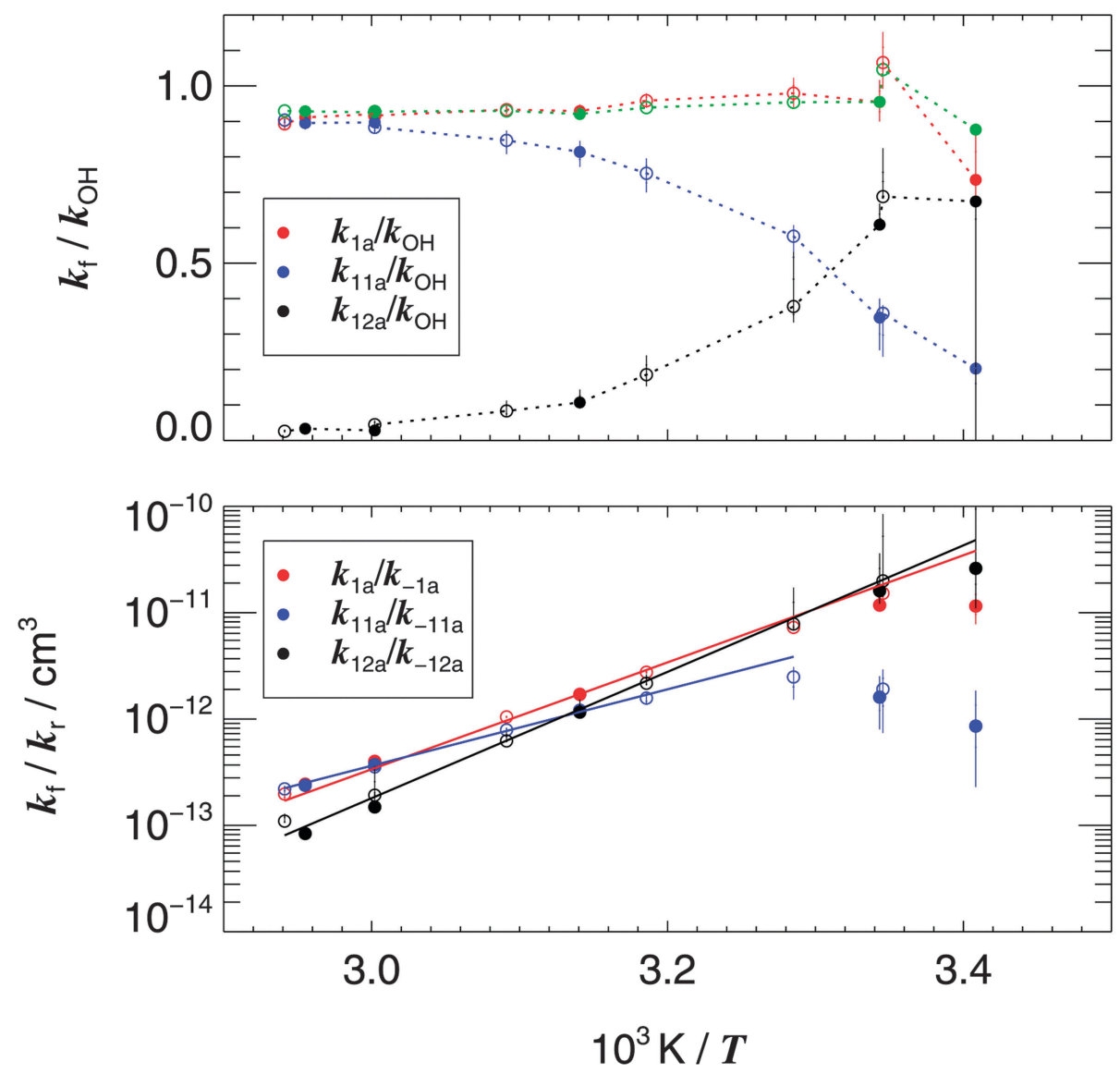

Fig. 3 Adduct yields (upper panel) and equilibrium constants (lower panel) of forward and reverse reaction rate constants of the OH $+1,3,5-$ TMB reactions using optimized adduct background loss rate constants from the $\mathrm{OH}$ balance (eqn (26) and (27)). Open symbols refer to measurements at 380 mbar and filled symbols at 750 mbar. Red: model-1, blue and black: model-2. Green points in the upper panel correspond to the total of blue and black points and dashed lines simply connect the data points. Full lines in the lower panel correspond to fitted, modified van't Hoff expressions (Table 6).

and consistent with the other background loss rate constants. The upper panel of Fig. 3 shows the obtained adduct yields. The yield for model-1 varies around 0.95 as expected because of the optimized $k_{3}$. The same applies for the total adduct yield of model-2 (green points). On the other hand, the yields of $\operatorname{add}_{1}$ and $\operatorname{add}_{2}$ clearly show an opposite and strongly changing

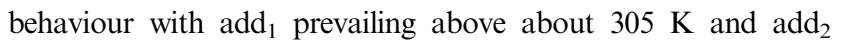
at lower temperatures. At the highest temperature of about $340 \mathrm{~K}$, $\operatorname{add}_{1}$ is formed almost exclusively. The same seems to be the case for $\operatorname{add}_{2}$ at the lowest temperatures, but the contributions at lower temperatures are rather uncertain because of the uncertainties of adduct background loss rate constants, in particular $k_{31}$. Despite these systematic uncertainties that depend on the interpretation of $k_{\text {add }}$, the error limits in Fig. 3 are relatively small because they result from the mutual dependencies of the originally fitted parameters rather than the error limits in Tables 1 and 2. In accordance with the correlation coefficients obtained in simulated experiments (Section S5.3, ESI $\dagger$ ) it was found that the maximum values of $k_{\mathrm{f}} k_{\mathrm{r}}$ always corresponded to maximum values of $k_{\mathrm{OH}}$ and $k_{\text {add }}$ and vice versa. Anyway, if the model-2 approach were correct and $\operatorname{add}_{2}$ were indeed the ipso-isomer, as speculated above, it would be a significant, or even the dominant product of the $\mathrm{OH}+1,3,5-\mathrm{TMB}$ reaction at room temperature and under atmospheric conditions.
To determine the stability of the adduct isomers we calculated the equilibrium constants $K_{\mathrm{c}}$ of the forward and reverse reactions. In the case of model-1:

$$
K_{\mathrm{c}}=\frac{k_{\mathrm{f}}}{k_{\mathrm{r}}}=\frac{k_{1 \mathrm{a}}}{k_{-1 \mathrm{a}}}=\frac{k_{1 \mathrm{a}} k_{-1 \mathrm{a}}}{k_{-1 \mathrm{a}}^{2}}=\frac{k_{1 \mathrm{a}} k_{-1 \mathrm{a}}}{\left(k_{\mathrm{add}}-k_{3}\right)^{2}}
$$

For model-2:

$$
\begin{aligned}
& K_{\mathrm{c} 1}=\frac{k_{11 \mathrm{a}}}{k_{-11 \mathrm{a}}}=\frac{k_{11 \mathrm{a}} k_{-11 \mathrm{a}}}{\left(k_{\mathrm{add}_{1}}-k_{31}\right)^{2}} \\
& K_{\mathrm{c} 2}=\frac{k_{12 \mathrm{a}}}{k_{-12 \mathrm{a}}}=\frac{k_{12 \mathrm{a}} k_{-12 \mathrm{a}}}{\left(k_{\mathrm{add}_{2}}-k_{32}\right)^{2}}
\end{aligned}
$$

Taking into account the reaction stoichiometry, $K_{\mathrm{c}}$ can be related to the standard reaction enthalpy $\Delta H_{\mathrm{r}, \mathrm{m}}^{\ominus}$ of adduct formation:

$$
\begin{aligned}
K_{\mathrm{c}} & =\frac{k_{\mathrm{f}}}{k_{\mathrm{r}}}=\frac{k T}{p^{\ominus}} \exp \left(-\Delta H_{\mathrm{r}, \mathrm{m}}^{\ominus} / R T+\Delta S_{\mathrm{r}, \mathrm{m}}^{\ominus} / R\right) \\
& \propto T \exp \left(-\Delta H_{\mathrm{r}, \mathrm{m}}^{\ominus} / R T\right)
\end{aligned}
$$

In this equation $\Delta S_{\mathrm{r}, \mathrm{m}}^{\ominus}$ is the standard reaction entropy, $k$ is the Boltzmann constant, $R$ is the gas constant and $p^{\ominus}$ the standard pressure. In a narrow temperature range this 
corresponds to an exponential dependence of $K_{\mathrm{c}}$ on temperature in good approximation. Nevertheless, we fitted the ratios to a modified Arrhenius, or in this case van't Hoff expression, $k_{\mathrm{f}} / k_{\mathrm{r}}=A_{4} T \exp \left(-B_{4} / T\right)$ according to eqn (31). For consistency reasons we used the same optimized $k_{3}, k_{31}$ and $k_{32}$ as before for the calculations of the adduct yields.

In the lower panel of Fig. 3 the $K_{\mathrm{c}}$ are plotted as a function of reciprocal temperatures, together with the fitted functions. For model-1 and $\mathrm{add}_{2}$ of model-2 the ratios show a linear behaviour in the semi-logarithmic plots. But in the case of $\operatorname{add}_{1}$ of model-2 the ratio drops strongly at low temperatures $(<300 \mathrm{~K})$. The fit was therefore confined to a temperature range $\geq 304 \mathrm{~K}$. If $k_{31}$ were increased to a value of about $8 \mathrm{~s}^{-1}$ as implied by the $C$-result of the empirical description in Table 4 , the ratios for $\operatorname{add}_{1}$ at low temperatures would be greater and more consistent with a linear dependence in Fig. 3 while the slope would approach that of model-1. However, as mentioned above, greater values of $k_{31}$ are inconsistent with the measured $k_{\mathrm{OH}}$.

The fitted parameters $A_{4}$ and $B_{4}$ are given in Table 6 together with the calculated reaction enthalpies and entropies. The results for model-1 again lie in between those of model-2. For model-2 the formation enthalpy of $\operatorname{add}_{1}$ of $-72 \pm 6 \mathrm{~kJ} \mathrm{~mol}^{-1}$ compares very favorably with a value of $-69 \pm 20 \mathrm{~kJ} \mathrm{~mol}^{-1}$ estimated by Perry et al. $^{26}$ for methyl-substituted benzenes including TMBs. Formation of $\mathrm{add}_{2}$ is significantly more exothermic: $-116 \pm 8 \mathrm{~kJ} \mathrm{~mol}^{-1}$. Theoretically greater reaction enthalpies were indeed predicted for ipso-type adducts by Uc et al. ${ }^{8}$ compared to ortho-, meta- and para-adducts in the case of toluene. However, the differences were minor and generally less than $20 \%$. In other theoretical studies orthoadducts were slightly favored energetically in the case of toluene $^{33,34}$ and $p$-xylene ${ }^{35}$ but the differences were again minor and significant yields of ipso-adducts were predicted in all cases. Andino and Vivier-Bunge ${ }^{36}$ give an overview on currently available theoretical studies on $\mathrm{OH}$-aromatic adduct isomers.

The main problem with the result for add $_{2}$ obtained here is that the negative reaction enthalpy is almost a factor of three greater than the activation energy for the reverse adduct dissociation (compare $B_{2}$ and $B_{4}$ in Tables 4 and 6) which is clearly inconsistent. Moreover, the reaction entropy for $\operatorname{add}_{2}$ formation is almost a factor of three greater than that for $\operatorname{add}_{1}$ and about a factor of two greater than a theoretically calculated value of $-101 \mathrm{~J} \mathrm{~mol}^{-1} \mathrm{~K}^{-1}$ for the $\mathrm{OH}$-benzene adduct $^{37}$ that should be in the same range. Taking also into account the unusually low factor $A_{2}$ for $\operatorname{add}_{2}$ (Table 4) and the deviations of $K_{\mathrm{c} 1}$ from the expected dependence at low temperatures (Fig. 3), we conclude that, despite the improved fit quality compared with model-1, also model-2 is inadequate.

\subsection{Model-3 with pure isomerization and intermediate cases}

To find out if an adduct isomerization can resolve the inconsistencies of model-2, fits to decay curves were also performed according to model-3 with the product $k_{12 \mathrm{a}} k_{-12 \mathrm{a}}$ set to zero. Consequently, a direct formation of $\mathrm{add}_{2}$ and its dissociation back to $\mathrm{OH}$ were deactivated while the product $k_{12} k_{21}$ was optimized. It turned out that the fitted curves and therefore also the fit qualities were identical to those of model-2. The same applies for the $k_{2}$ and the $k_{\mathrm{OH}}$ that we also identical. Thus from the $\mathrm{OH}$ decays alone we are unable to distinguish between an isomerization $\operatorname{add}_{1} \rightarrow \operatorname{add}_{2}$ and a direct formation $\mathrm{OH}+$ aromatic $\rightarrow \operatorname{add}_{2}$. Consequently, a full fit where all seven rate constant related parameters of model-3 are optimized simultaneously was not sensible because any intermediate case between the two extremes, i.e. $k_{12 \mathrm{a}} k_{-12 \mathrm{a}}=0$ as assumed here and $k_{12} k_{21}=0$ (model-2), would also explain the $\mathrm{OH}$ decays. This can be rationalized in terms of the parameters $s, t$ and $u$ in eqn (9), (21) and (22) that determine

Table 7 Model-3 fit results and estimated error limits from simultaneous triexponential fits to $m$ decay curves at different $1,3,5-\mathrm{TMB}$ concentrations (see Table 1 for $m$ and experimental conditions). The parameters $k_{2}, k_{11 \mathrm{a}}+k_{12 \mathrm{a}}+k_{1 \mathrm{~b}}, \mathrm{DOF}$ and $\chi^{2} / \mathrm{DOF}$ are identical to those in Table 2 . The product $k_{12 \mathrm{a}} k_{-12 \mathrm{a}}$ was set to zero

\begin{tabular}{|c|c|c|c|c|}
\hline \# & $\begin{array}{l}k_{111} k_{-11 \mathrm{a}} / \\
10^{-10} \mathrm{~cm}^{3} \mathrm{~s}^{-2}\end{array}$ & $\begin{array}{l}k_{-11 \mathrm{a}}+k_{12} \\
+k_{31} / \mathrm{s}^{-1}\end{array}$ & $k_{21}+k_{32} / \mathrm{s}^{-1}$ & $k_{12} k_{21} / \mathrm{s}^{-2}$ \\
\hline 4 & $2.5 \pm{ }_{0.55}^{0.88}$ & $12.6 \pm{ }_{4.5}^{11}$ & $6.6 \pm{ }_{4.3}^{9.6}$ & $30 \pm{ }_{26}^{159}$ \\
\hline 5 & $3.2 \pm 0.57$ & $10.1 \pm 2.1$ & $5.9 \pm \begin{array}{l}6.0 \\
3.0\end{array}$ & $14 \pm{ }_{11}^{48}$ \\
\hline 6 & $3.5 \pm 0.58$ & $11.7 \pm 2.9$ & $6.3 \pm \begin{array}{l}4.3 \\
2.8\end{array}$ & $18 \pm 39$ \\
\hline 7 & $5.1 \pm 0.60$ & $13.5 \pm 2.6$ & $5.7 \pm \begin{array}{l}4.1 \\
2.5\end{array}$ & $11 \pm \frac{22}{7}$ \\
\hline 8 & $11.2 \pm{ }_{0.86}^{1.0}$ & $26.4 \pm 2.3$ & $7.2 \pm 2.7 .0$ & $17 \pm{ }_{8}^{16}$ \\
\hline 9 & $18.2 \pm \begin{array}{l}2.3 \\
1.9\end{array}$ & $39.0 \pm 3.7$ & $7.5 \pm 3.4$ & $18 \pm{ }_{9}^{21}$ \\
\hline 10 & $27.3 \pm 4.9$ & $59.0 \pm 5.4$ & $9.6 \pm{ }_{3.1}^{4.4}$ & $30 \pm{ }_{16}^{36}$ \\
\hline 11 & $55.1 \pm 17$ & $125 \pm 14$ & $13.7 \pm 6.3$ & $56 \pm{ }_{31}^{70}$ \\
\hline 12 & $50.1 \pm{ }_{13}^{20}$ & $117 \pm \frac{22}{15}$ & $11.0 \pm \begin{array}{l}14 \\
6.7\end{array}$ & $26 \pm{ }_{21}^{116}$ \\
\hline 13 & $99.6 \pm 25$ & $205 \pm 29$ & $23.8 \pm 11$ & $124 \pm 75$ \\
\hline 14 & $107 \pm 33$ & $221 \pm{ }_{29}^{34}$ & $14.7 \pm 7.0$ & $67 \pm 36$ \\
\hline
\end{tabular}

Table 6 Parameters $A_{4}$ and $B_{4}$ from fitted, modified van't Hoff expressions $k_{\mathrm{f}} / k_{\mathrm{r}}=A_{4} T$ exp $\left(-B_{4} / T\right)$ to ratios of forward and reverse rate constants $k_{\mathrm{f}} / k_{\mathrm{r}}$, standard reaction enthalpies $\Delta H_{\mathrm{r}, \mathrm{m}}^{\ominus}$, and reaction entropies $\Delta S_{\mathrm{r}, \mathrm{m}}^{\ominus}$ for the adduct formation reactions for $1,3,5$-TMB. For model-1: $k_{\mathrm{f}} / k_{\mathrm{r}}=k_{1 \mathrm{a}} / k_{-1 \mathrm{a}}$. For model-2: $k_{\mathrm{f}} / k_{\mathrm{r}}=k_{11 \mathrm{a}} / k_{-11 \mathrm{a}}\left(\operatorname{add}_{1}\right)$ and $k_{\mathrm{f}} / k_{\mathrm{r}}=k_{12 \mathrm{a}} / k_{-12 \mathrm{a}}\left(\operatorname{add}_{2}\right)$. For $\operatorname{model}-3: k_{\mathrm{f}} / k_{\mathrm{r}}=k_{11 \mathrm{a}} / k_{-11 \mathrm{a}}\left(\operatorname{add}{ }_{1}\right)$ and $k_{\mathrm{f}} / k_{\mathrm{r}}=k_{12} / k_{21}\left(\operatorname{add}_{1} \rightleftharpoons \operatorname{add}_{2}\right)$. Reaction enthalpies correspond to the product $R B_{4}$, reaction entropies to the product $R \ln (7.242 \times$ $10^{21} \mathrm{~cm}^{-3} \mathrm{~K}_{4}$ )

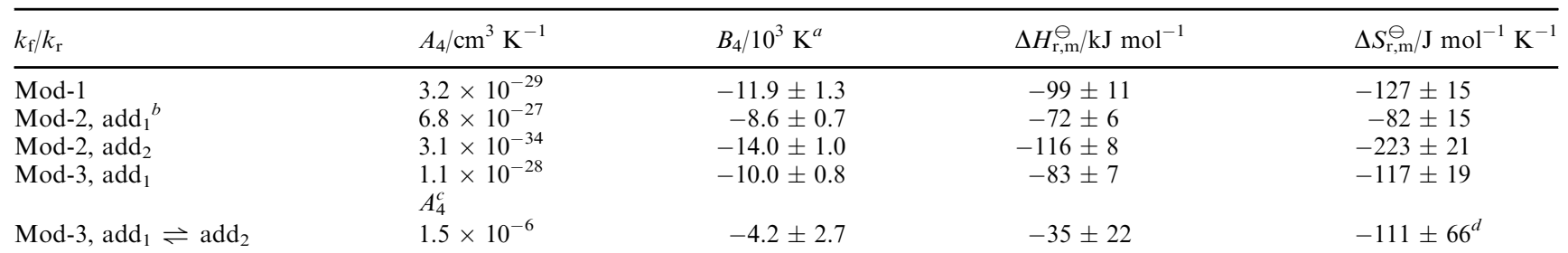

${ }^{a}$ Error limit estimated from fits to maximum and minimum $k_{\mathrm{f}} / k_{\mathrm{r}} \cdot{ }^{b} T \geq 304 \mathrm{~K} .{ }^{c}$ Dimensionless, fit to normal exponential van't Hoff expression.

${ }^{d}$ Calculated from the product $R \ln \left(A_{4}\right)$. 
the same optimized decay curves. Except for $a=k_{2}+k_{\mathrm{OH}}$, the quantities $d+g=k_{\mathrm{add}_{1}}+k_{\mathrm{add}_{2}},(b c+e f) /[$ aromatic] $=$ $k_{11 \mathrm{a}} k_{-11 \mathrm{a}}+k_{12 \mathrm{a}} k_{-12 \mathrm{a}}$ and $d g-h i=k_{\mathrm{add}_{1}} k_{\mathrm{add}_{2}}-k_{21} k_{12}$ are constant as can be verified by comparison of the data in Tables 2 and 7.

In Table 7 the fit parameters that were different for model-3 are listed. These results are also plotted in Fig. 4 as a function of reciprocal temperatures in comparison with those obtained with model-2. The differences for the two $k_{\text {add }}$ and $k_{11 a} k_{-11 \text { a }}$ were minor which is also reflected in the similar empirical parameters $A_{2}, B_{2}$ and $A_{3}, B_{3}$ in
Tables 4 and 5, respectively. However, the interpretation of the adduct loss rate constants is different here: $k_{\text {add }_{1}}=k_{-11 \mathrm{a}}+$ $k_{12}+k_{31}$ and $k_{\mathrm{add}_{2}}=k_{21}+k_{32}$.

We again determined optimized $k_{31}, k_{32}$ and the total $k_{12}+k_{31}$ by consulting the $\mathrm{OH}$ reaction balance similar to eqn (27):

$$
\begin{aligned}
& k_{11 \mathrm{a}}=k_{\mathrm{OH}}-k_{1 \mathrm{~b}}=\frac{k_{11 \mathrm{a}} k_{-11 \mathrm{a}}}{k_{\mathrm{add}_{1}}-k_{12}-k_{31}} \\
& =\frac{k_{11 \mathrm{a}} k_{-11 \mathrm{a}}\left(k_{\mathrm{add}_{2}}-k_{32}\right)}{\left(k_{\mathrm{add}_{1}}-k_{31}\right)\left(k_{\mathrm{add}_{2}}-k_{32}\right)-k_{12} k_{21}}
\end{aligned}
$$
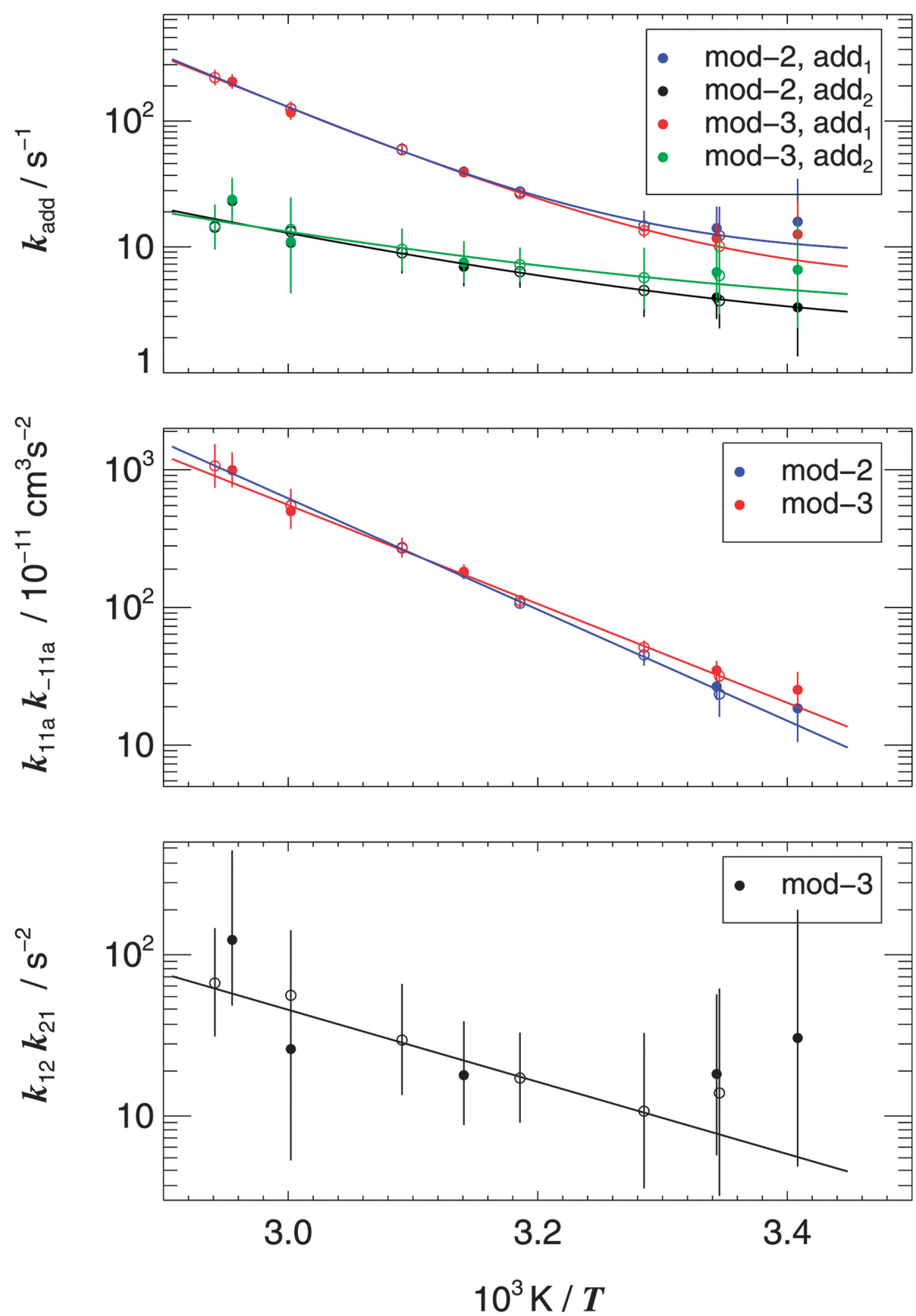

Fig. 4 Rate constant related fit parameters for 1,3,5-TMB from model-2 (blue, black) and model-3 (red, green). Open symbols refer to measurements at $380 \mathrm{mbar}$ and filled symbols at $750 \mathrm{mbar}$. Full lines correspond to fitted Arrhenius expressions (Tables 4 and 5). In the first panel temperature independent contributions of background loss rate constants were assumed. Black data points in the third panel correspond to model-3. 
$k_{31}=0.6 \mathrm{~s}^{-1}, k_{32}=2.7 \mathrm{~s}^{-1}$ and $k_{12}+k_{31}=5.1 \mathrm{~s}^{-1}$ were obtained this way. The latter two rate constants are in good agreement with the empirical parameters $C$ in Table 4 .

With these optimized rate constants, equilibrium constants were calculated for the formation of $\mathrm{add}_{1}$ in the $\mathrm{OH}$ reaction,

$$
K_{\mathrm{c} 1}=\frac{k_{11 \mathrm{a}}}{k_{-11 \mathrm{a}}}=\frac{k_{11 \mathrm{a}} k_{-11 \mathrm{a}}}{\left(k_{\mathrm{add}_{1}}-k_{12}-k_{31}\right)^{2}}
$$

and for formation of $\operatorname{add}_{2}$ by isomerization:

$$
K_{\mathrm{c} 2}=\frac{k_{12}}{k_{21}}=\frac{k_{12} k_{21}}{\left(k_{\mathrm{add}_{2}}-k_{32}\right)^{2}}
$$

The $K_{\mathrm{c} 1}$ were then evaluated as before according to eqn (31). The corresponding plot is shown in the upper panel of Fig. 5. Expectedly, the resulting reaction enthalpy of $-83 \pm 7 \mathrm{~kJ} \mathrm{~mol}^{-1}$ is similar to that obtained with model-2 within the combined error limits (Table 6) whilst the reaction entropies are slightly different and are both in fair agreement with the theoretical result for benzene. ${ }^{37}$

For the $K_{\mathrm{c} 2}$ the factor $k T / p^{\ominus}$ in eqn (31) does not apply because of the 1:1 stoichiometry of the isomerization reaction. A normal van't Hoff exponential fit was therefore made to obtain the reaction enthalpy of the isomerization $\operatorname{add}_{1} \rightarrow \operatorname{add}_{2}$ as shown in the lower panel of Fig. 5. The corresponding value is $-35 \pm 22 \mathrm{~kJ} \mathrm{~mol}^{-1}$. From this result, together with that for the formation of $\operatorname{add}_{1}$, a reaction enthalpy of $-120 \pm 30 \mathrm{~kJ} \mathrm{~mol}^{-1}$ was calculated for $\mathrm{add}_{2}$ formation. Except for the greater uncertainty, that was estimated conservatively, this enthalpy is also the same as from model-2 although the underlying reaction mechanism is different. We maintain the assumption that the more stable add ${ }_{2}$ corresponds to the ipso-isomer.

The reaction entropy for the isomerization is smaller than the theoretically expected value that is close to zero. Nevertheless, the overall result of model-3 is more consistent than that of model-2 because the relatively small value of parameter $B_{2}\left(\operatorname{add}_{2}\right.$ loss rate constant) now corresponds to the activation energy of the isomerization rather than of adduct dissociation. Moreover, also the low preexponential factor is explainable for the isomerization if the reaction proceeds on a non-adiabatic pathway. To our knowledge the isomerization reactions have not yet been evaluated in detail theoretically but Uc et al. ${ }^{8}$ mention the possibility and a potential catalysis by $\mathrm{O}_{2}$. Andino and Vivier-Bunge ${ }^{36}$ indicate the existence of a high activation energy of about $90 \mathrm{~kJ} \mathrm{~mol}^{-1}$ for the ipso $\rightarrow$ ortho-isomerization that is in qualitative agreement with our result. On the other hand, the results obtained here with model-3 imply that the ortho $\rightarrow$ ipso-isomerization is a slow process $\left(\approx 4 \mathrm{~s}^{-1}\right)$ with little if any activation energy.
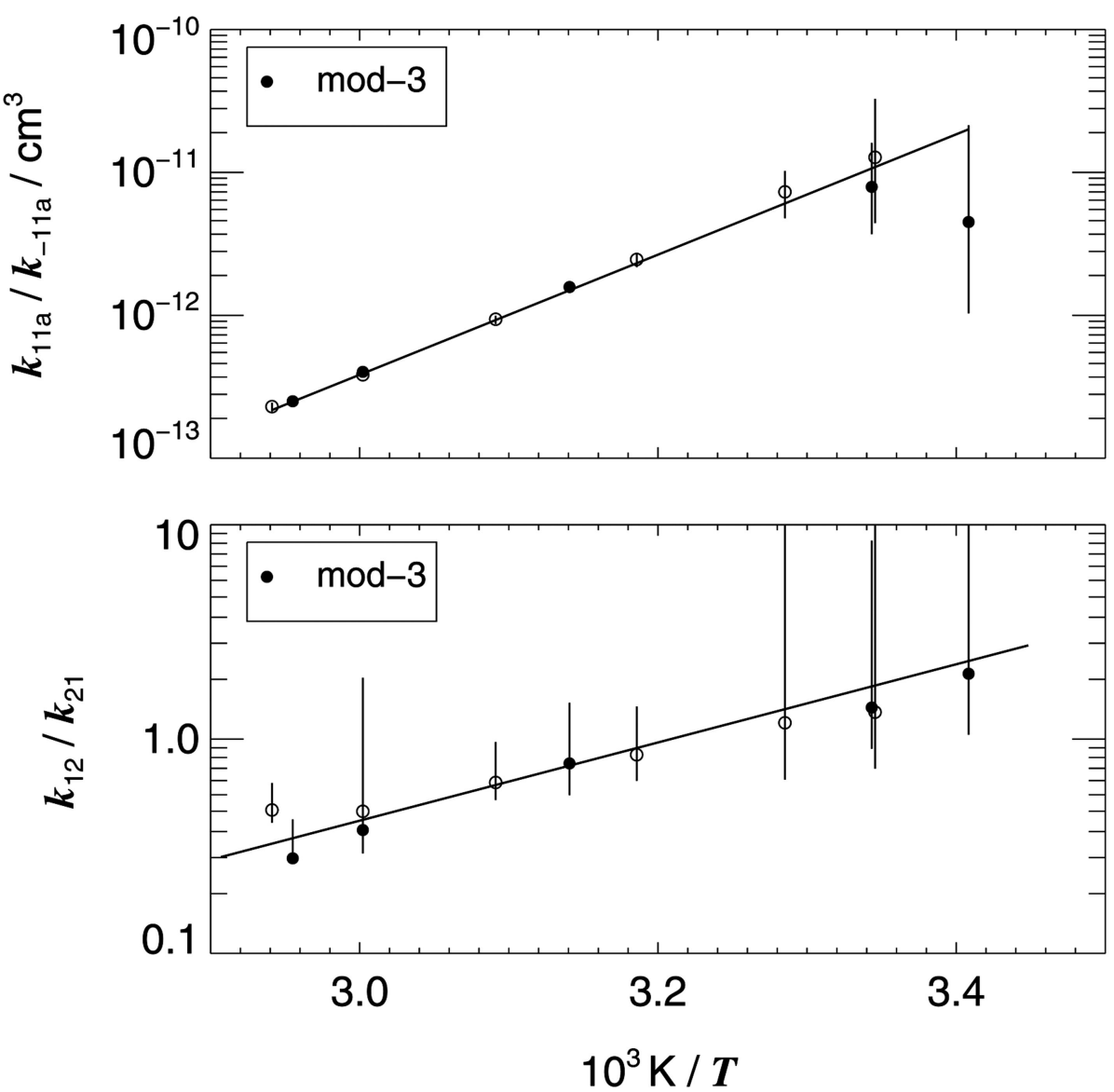

Fig. 5 Equilibrium constants for the $\mathrm{OH}+1,3,5-\mathrm{TMB}$ reaction and adduct isomerization from model-3. Open symbols refer to measurements at $380 \mathrm{mbar}$ and filled symbols at $750 \mathrm{mbar}$. Full lines are fits to a modified van't Hoff expression (upper panel) and a normal van't Hoff expression (lower panel). The corresponding parameters can be found in Table 6 . 
Presumably, the true reaction mechanism is a mixture of the limiting cases discussed so far. A slow, underlying dissociation of $\operatorname{add}_{2}$ back to $\mathrm{OH}+$ aromatic, e.g. similar to that observed for $\mathrm{HMB},{ }^{29}$ would leave room for a direct formation of $\mathrm{add}_{2}$ in the $\mathrm{OH}$ reaction. This is very likely, given the fast reaction of $\mathrm{OH}$ with $\mathrm{HMB}$ determined by Berndt and Böge. ${ }^{9}$ Moreover, a contribution of adduct dissociation to $k_{\mathrm{add}_{2}}$ would weaken the decrease of the equilibrium constant $K_{\mathrm{c} 2}$ towards higher temperatures (Fig. 5) thereby decreasing the reaction enthalpy and increasing the reaction entropy of the isomerization towards the theoretically expected value. In addition, this would lead to greater values of $k_{12}$ with increasing temperature which means that probably both isomerization reactions are slow processes with low activation energies. That points towards a complex mechanism, e.g. via cyclic epoxy-type intermediates.

It should be noted that also catalyzed isomerizations of the type:

$$
\operatorname{add}_{1}+\operatorname{aromatic} \rightleftharpoons \operatorname{add}_{2}+\text { aromatic }
$$

could be operative. These reactions would produce a different aromatic concentration dependence compared to model-3 and were included in the analytical treatment for two selected temperatures. However, no improvement of fit qualities was achieved by applying a corresponding dependence of the parameter $h i$ on [aromatic]. Thus, we find no indication that these exchange reactions are of importance under our experimental conditions.

Based on the available data of this work, obviously no final conclusion can be drawn regarding the primary yields of the adducts in the $\mathrm{OH}$ reaction. This is unfortunate because for the atmospheric degradation of $1,3,5-\mathrm{TMB}$ these primary yields are probably important. The reason is that neither the dissociation of the adducts back to $\mathrm{OH}$ nor an isomerization with rate constants as estimated in this work, i.e. $\leq 5 \mathrm{~s}^{-1}$, can compete with the fast secondary reaction of the adducts with $\mathrm{O}_{2}$ under atmospheric conditions. On the other hand, the oxygen itself could influence the isomerization and atmospheric product yields. Clearly more work is needed to elucidate the details of the mechanism. Theoretical calculations could help to clarify the discrepancy between the apparently low yield of the ipso-isomer consistently obtained here with model-2 and model-3 at temperatures $>310 \mathrm{~K}$ and the fast rate constant of $\mathrm{OH}$ reaction with hexamethylbenzene. High quality measurements with other compounds that are expected to behave similarly, e.g. 1,2,4,5-tetramethylbenzene could lead to more conclusive results. Finally there may be other, more direct methods to determine the primary yields of the adduct isomers.

\section{Conclusions}

$\mathrm{OH}$ decay curves in the presence of 1,3,5-trimethylbenzene were analysed in terms of a reaction model with reversible formation of $\mathrm{OH}$-aromatic adducts leading to slow regeneration of $\mathrm{OH}$. In contrast to previous studies, we examined the possibility of formation of two adduct isomers, namely orthoand ipso-type $\mathrm{OH}$-aromatic adducts as predicted theoretically. The mechanism was extended accordingly and analytical solutions were derived to fit the corresponding triexponential
$\mathrm{OH}$ decay curves. Compared to the previous approach with only one adduct species and biexponential decay curves, the extended mechanism led to significant improvements of fit qualities, supporting formation of two adduct isomers. Moreover, the $\mathrm{OH}$ rate constants were different and in better agreement with literature data. Similar differences were found for the rate constants of $\mathrm{OH}+1,2,3$-trimethylbenzene and 1,2,4-trimethylbenzene. The results based on the extended mechanism are therefore preferred for all three aromatic compounds and corresponding Arrhenius expressions were derived.

Formation of adduct isomers was studied in detail for 1,3,5trimethylbenzene. The apparent kinetic properties of the adduct isomers were strongly different but the calculated thermochemical data for formation and dissociation of the more stable adduct isomer were contradictory. An alternative mechanism was therefore tested where the second isomer is formed by isomerization instead of the $\mathrm{OH}$ reaction. This led to the same fit functions but more consistent thermochemical results with reaction enthalpies of $-83 \pm 7 \mathrm{~kJ} \mathrm{~mol}^{-1}$ and $-35 \pm 22 \mathrm{~kJ} \mathrm{~mol}^{-1}$ for the formation of one adduct isomer and the isomerization into the other, respectively. Based on a comparison with literature results for benzene and hexamethylbenzene, the more stable adduct was assigned to the ipso-adduct. An intermediate mechanism with formation of both adducts by $\mathrm{OH}$ reaction and isomerization is likely but cannot be further specified based on the available data.

\section{Acknowledgements}

This work was supported within the joint French-German research project ATMOCHEM funded by the Deutsche Forschungsgemeinschaft under grant BO-1580/3-1 and ZE 792/6-1. We thank R. Koch for detecting the triexponential feature of the decays of $\mathrm{OH}$ in the presence of 1,3,5-trimethylbenzene, M. Elend for the GC-analysis of the liquid reactants, and R. Koch and M. Siese for their efforts to improve the instrument.

\section{References}

1 J. G. Calvert, R. Atkinson, K. H. Becker, R. M. Kamens, J. H. Seinfeld, T. J. Wallington and G. Yarwood, Mechanisms of atmospheric oxidation of aromatic hydrocarbons, Oxford University Press, 2002.

2 R. Atkinson and J. Arey, Chem. Rev., 2003, 103, 4605-4638.

3 R. Atkinson and J. Arey, Polycyclic Aromat. Compd., 2007, 27, 15-40.

4 A. Wahner and C. Zetzsch, J. Phys. Chem., 1983, 87, 4945-4951.

5 F. Witte, E. Urbanik and C. Zetzsch, J. Phys. Chem., 1986, 90, 3251-3259.

6 R. Knispel, R. Koch, M. Siese and C. Zetzsch, Ber. Bunsen-Ges., 1990, 94, 1375-1379.

7 R. Koch, R. Knispel, M. Elend, M. Siese and C. Zetzsch, Atmos. Chem. Phys., 2007, 7, 2057-2071.

8 V. H. Uc, I. Garcia-Cruz, A. Hernandez-Laguna and A. VivierBunge, J. Phys. Chem. A, 2000, 104, 7847-7855.

9 T. Berndt and O. Böge, Int. J. Chem. Kinet., 2001, 33, 124-129.

10 H. Geiger, I. Barnes, K. H. Becker, B. Bohn, T. Brauers, B. Donner, H. P. Dorn, M. Elend, C. M. F. Dinis, D. Grossmann, H. Hass, H. Hein, A. Hoffmann, L. Hoppe, F. Hülsemann, D. Kley, B. Klotz, H. G. Libuda, T. Maurer, D. Mihelcic, G. Moortgat, R. Olariu, P. Neeb, D. Poppe, L. Ruppert, C. G. Sauer, O. Shestakov, H. Somnitz, W. R. Stockwell, L. P. Thüner, A. Wahner, P. Wiesen, F. Zabel, R. Zellner and C. Zetzsch, J. Atmos. Chem., 2002, 42, 323-357. 
11 F. Stuhl and H. Niki, J. Chem. Phys., 1972, 57, 3671-3677.

12 F. Stuhl and H. Niki, J. Chem. Phys., 1972, 57, 3677-3679.

13 R. Koch, PhD thesis, University of Hannover, 1992.

14 R. Knispel, PhD thesis, University of Hannover, 1993.

15 E. R. Hopke and G. W. Sears, J. Am. Chem. Soc., 1948, 70, 3801-3803.

16 R. M. Stephenson, S. Malanowski and D. Ambrose, Handbook of the thermodynamics of organic compounds, Elsevier, 1987.

17 S. Zhang and C. Zetzsch, Gas Kinetics Conference 2010, 2010.

18 S. P. Sander, J. Abbatt, J. R. Barker, J. B. Burkholder, R. R. Friedl, D. M. Golden, R. E. Huie, C. E. Kolb, M. J. Kurylo, G. K. Moortgat, V. L. Orkin and P. H. Wine, Chemical kinetics and photochemical data for use in atmospheric studies, Evaluation No. 17, Jet Propulsion Laboratory, Pasadena, 2011 http://jpldataeval. jpl.nasa.gov, 2011.

19 A. Bolovinos, J. Philis, E. Pantos, P. Tsekeris and G. Andritsopoulos, J. Chem. Phys., 1981, 75, 4343-4349.

20 A. Bolovinos, J. Philis, E. Pantos, P. Tsekeris and G. Andritsopoulos, J. Mol. Spectrosc., 1982, 94, 55-68.

21 R. A. Alberty, J. Chem. Educ., 2004, 81, 1206-1209.

22 R. Atkinson, J. Phys. Chem. Ref. Data, 1989, Monograph 1204.

23 C. Markwardt, IDL Library, http://cow.physics.wisc.edu/ craigm/idl/, 2010.

24 S. Nehr, B. Bohn, H. Fuchs, A. Hofzumahaus and A. Wahner, Phys. Chem. Chem. Phys., 2011, 13, 10699-10708.

25 D. A. Hansen, R. Atkinson and J. N. Pitts, J. Phys. Chem., 1975, 79, 1763-1766.

26 R. A. Perry, R. Atkinson and J. N. Pitts, J. Phys. Chem., 1977, 81, 296-304.
27 S. M. Aschmann, W. D. Long and R. Atkinson, J. Phys. Chem. A, 2006, 110, 7393-7400.

28 M. J. Pilling and P. W. Seakins, Reaction Kinetics, Oxford University Press, 1996.

29 J. von Buttlar, R. Koch, M. Siese and C. Zetzsch, EGU General Assembly 2008, Geophys. Res. Abstr. 10, 2008, EGU2008-A-10576.

30 F. P. Tully, A. R. Ravishankara, R. L. Thompson, J. M. Nicovich, R. C. Shah, N. M. Kreutter and P. H. Wine, J. Phys. Chem., 1981, 85, 2262-2269.

31 J. M. Nicovich, R. L. Thompson and A. R. Ravishankara, J. Phys. Chem., 1981, 85, 2913-2916.

32 IUPAC Subcommittee on gas kinetic data evaluation, http://www. iupac-kinetic.ch.cam.ac.uk/, 2008.

33 I. Suh, D. Zhang, R. Zhang, L. T. Molina and M. J. Molina, Chem. Phys. Lett., 2002, 364, 454-462.

34 D. Johnson, S. Raoult, R. Lesclaux and L. N. Krasnoperov, J. Photochem. Photobiol., A, 2005, 176, 98-106.

35 J. Fan and R. Zhang, J. Phys. Chem. A, 2006, 110, 7728-7737.

36 J. M. Andino and A. Vivier-Bunge, Adv. Quantum Chem., 2008, 55, 297-310.

37 T. H. Lay, J. W. Bozzelli and J. H. Seinfeld, J. Phys. Chem., 1996, 100, 6543-6554.

38 R. Atkinson, S. Aschmann, J. Arey and W. Carter, Int. J. Chem. Kinet., 1989, 21, 801-827.

39 F. Kramp and S. E. Paulson, J. Phys. Chem. A, 1998, 102, 2685-2690.

40 S. M. Aschmann, E. C. Tuazon and R. Atkinson, J. Phys. Chem. A, 2005, 109, 2282-2291. 